\title{
On particle acceleration and transport in plasmas in the Galaxy: theory and observations
}

\author{
Elena Amato ${ }^{(1,2, \dagger}$ and Sabrina Casanova ${ }^{3,4}$ \\ ${ }^{1}$ INAF - Osservatorio Astrofisico di Arcetri, Largo E. Fermi, 5, 50125, Firenze, Italy \\ ${ }^{2}$ Università degli Studi di Firenze, Via Sansone 1, 50019, Sesto Fiorentino (FI), Italy \\ ${ }^{3}$ Institute of Nuclear Physics PAN, Radzikowskiego 152, 31-342 Kraków, Poland \\ ${ }^{4}$ Max Planck Institute for Nuclear Physics, Saupfercheckweg 1, 69117, Heidelberg, Germany
}

(Received 23 October 2020; revised 13 January 2021; accepted 13 January 2021)

Accelerated particles are ubiquitous in the Cosmos and play a fundamental role in many processes governing the evolution of the Universe at all scales, from the sub-AU scale relevant for the formation and evolution of stars and planets to the Mpc scale involved in Galaxy assembly. We reveal the presence of energetic particles in many classes of astrophysical sources thanks to their production of non-thermal radiation, and we detect them directly at the Earth as cosmic rays. In the last two decades both direct and indirect observations have provided us a wealth of new, high-quality data about cosmic rays and their interactions both in sources and during propagation, in the Galaxy and in the Solar System. Some of the new data have confirmed existing theories about particle acceleration and propagation and their interplay with the environment in which they occur. Some others have brought about interesting surprises, whose interpretation is not straightforward within the standard framework and may require a change of paradigm in terms of our ideas about the origin of cosmic rays of different species or in different energy ranges. In this article, we focus on cosmic rays of galactic origin, namely with energies below a few petaelectronvolts, where a steepening is observed in the spectrum of energetic particles detected at the Earth. We review the recent observational findings and the current status of the theory about the origin and propagation of galactic cosmic rays.

Key words: astrophysical plasmas, plasma instabilities, plasma nonlinear phenomena

\section{Introduction}

Cosmic rays (CRs) have been known and studied for more than a century now (see e.g. Amato (2014) and Blasi (2013) for recent reviews). They are highly energetic charged particles, mainly protons and He nuclei, with a minor fraction of heavier nuclei (1\%), electrons $(2 \%)$ and anti-matter particles (positrons and anti-protons, 1\%o). Their origin was associated with supernova (SN) explosions in the Galaxy already in the 1930s and the mechanism by which they would be accelerated up to very high energies (VHEs) in the blast waves emerging from SN explosions was proposed in the late 1970s (see § 2).

$\dagger$ Email address for correspondence: amato@arcetri.astro.it 
However, no direct proof of this association has yet been found and, as we discuss in the following, some recent developments, in both theory and high-energy astrophysical observations, have actually cast doubt on whether supernova remnants (SNRs) should be considered as the primary $\mathrm{CR}$ accelerators in the Galaxy throughout the entire energy range, up to $\mathrm{PeV}\left(10^{15} \mathrm{eV}\right)$ energies. At the same time, direct $\mathrm{CR}$ measurements have shown that particle transport is more complex than traditionally considered, and that additional sources are likely needed at least for CR positrons (see e.g. Amato \& Blasi (2018) for a recent review, and $\S 6$ for more details).

In recent years it has become increasingly clear that understanding the processes that govern the acceleration and transport of energetic particles is a key to answering a number of unsettled questions in astrophysics. Energetic particles, which we often refer to as CRs in the following, play a role in many astrophysical systems. To mention just a few, from the smallest to the largest scales, CRs affect the behaviour of planetary magnetospheres (Grießmeier et al. 2015), they are believed to be the main ionising agents penetrating dense gas clouds and regulating star formation (Padovani et al. 2020), they are suspected to be the primary drivers of galactic winds and the rulers of galactic feedback (Buck et al. 2020), and might even have a role in the generation of intergalactic magnetic fields (Donnert et al. 2018). In some of these situations, the interaction between CRs and the ambient plasma is simply described in terms of momentum exchange, in some others the nature of CRs as charged particles, carrying an electric current, is instead fundamental. In all cases, the correct description of the interaction between CRs and the ambient medium is an extremely challenging plasma physics problem (see e.g. Zweibel 2013).

All the information we can use to try to understand this problem comes from two kinds of observations: direct detection of CRs at Earth and detection of non-thermal radiation from astrophysical sources. In the last two decades, both types of observations have experienced enormous developments.

In terms of indirect detection, most of the news have come from high-energy telescopes observing the sky in the X-ray and $\gamma$-ray bands. The launch of Chandra was quickly followed by two important results in terms of CR physics. First, the detection of non-thermal $\mathrm{keV}$ photons from SNRs highlighted the presence of multi-TeV electrons in these sources: because in most of the acceleration mechanisms considered in astrophysics, and certainly in that proposed for SNRs, the acceleration process only depends on particle rigidity, this was also taken as indirect evidence of the presence of multi-TeV hadrons in SNRs. In addition, the excellent spatial resolution of Chandra allowed the region of non-thermal X-ray emission to be resolved, showing it to be very thin, with a typical size of few \% of pc (see Vink (2012) for a review). Such a small size, when interpreted as due to synchrotron energy losses of the emitting electrons, indicates the presence of a magnetic field of several $100 \mu \mathrm{G}$ downstream of the SNR shock. Such a large value of the magnetic field cannot simply result from shock compression of the typical interstellar field $\left(B_{\text {ISM }} \approx 3 \mu \mathrm{G}\right)$, but rather requires an extremely efficient amplification process to be at work.

At the same time as these discoveries, theory was also taking a major step forward with the identification of a mechanism through which CRs could provide magnetic field amplification (MFA) well beyond the quasi-linear theory limit of $\delta B / B \approx 1$. The process invoked is the non-resonant streaming instability (Bell 2004), which we discuss further in $\S 3$ : this is a current-induced instability that operates in situations where the CR energy density dominates over that of the background magnetic field. This condition is easily realised in young, rapidly expanding SNRs, if CRs are accelerated with efficiency of the order of $10 \%$, as required by the SNR-CR connection paradigm. The X-ray observations showing evidence for amplified magnetic fields were then taken as a proof 
of this connection: the amplified magnetic field implied that efficient CR acceleration was ongoing, and provided, at the same time, the ideal conditions for CRs to reach VHEs.

At higher photon energies, the $\gamma$-ray satellites AGILE and Fermi, observing the sky in the $20 \mathrm{MeV}-300 \mathrm{GeV}$ energy range, and the Cherenkov detectors H.E.S.S., VERITAS and MAGIC operating between tens of $\mathrm{GeV}$ and $\sim 10 \mathrm{TeV}$, were providing, in the meantime, interesting but somewhat surprising data. For the first time, direct evidence of relativistic protons was found in two middle-aged ( $~ 20-30 \mathrm{kyr}$ old) SNRs interacting with molecular clouds, W44 (Abdo et al. 2010a; Giuliani et al. 2011) and IC443 (Abdo et al. 2010b; Tavani et al. 2010). These sources were not expected to be efficient CR accelerators and indeed the proton spectrum inferred from observations is cut off at relatively low energies, $\sim 10 \mathrm{GeV}$ in W44, $\sim 10 \mathrm{TeV}$ in IC443. Follow-up theoretical studies showed that, in fact, at least in the case of W44, we are likely not witnessing fresh acceleration of CRs, with particles directly extracted from the interstellar plasma crossing the shock, but rather re-acceleration of particles from the galactic CR pool (e.g. Uchiyama et al. 2010; Cardillo, Amato \& Blasi 2016). As far as young sources are concerned, $\gamma$-ray observations have been partly disappointing, failing to provide indisputable evidence of hadronic acceleration (Funk 2015), and often showing steeper spectra than theoretically predicted in the case of efficient acceleration (Amato 2014). We discuss $\gamma$-ray observations of SNRs and the implications of these findings on CR acceleration in SNRs in $\S \S 2$ and 3 , respectively.

In terms of direct detection, experiments such as PAMELA, AMS-02 and CREAM have provided us with very precise measurements of the CR spectrum and composition up to $\mathrm{TeV}$ energies. Among the many discoveries, the most important in terms of implications on our understanding of CR acceleration and transport are: (1) the detection of a hardening in the spectra of protons, He nuclei and virtually all primary nuclei (Ahn et al. 2010; Adriani et al. 2011; Aguilar et al. 2015a,b), at $R \approx 300 \mathrm{GV}$, where $R=c p / Z e$ is the particle rigidity, with $p$ the particle momentum, $Z$ its atomic number, $e$ the electron charge and $c$ the speed of light; (2) the hardening found in the spectrum of secondary CRs at $R \approx 200 \mathrm{GV}$, with a change of spectral slope that is approximately twice as large as that of primaries (Aguilar et al. 2018); (3) the energy dependence of the ratio between secondary and primary CRs, that has now been measured with excellent statistics up to TV rigidities (Aguilar et al. 2016b) and provides invaluable constraints on the energy dependence of particle transport in the Galaxy; (4) a rise in the fraction of positrons-to-electrons at energies larger than $30 \mathrm{GeV}$ (Adriani et al. 2009; Aguilar et al. 2013), possibly suggesting the presence of an additional source of positrons in the Galaxy; (5) the spectrum of anti-p which is unexpectedly very close to that of protons and positrons (Aguilar et al. 2016a). We discuss these discoveries and their implications for the origin of CRs in $\S 4$.

\section{Testing the SNR paradigm}

While as early as the 1930s Baade \& Zwicky (1934) suggested that CRs might originate from SNs, the SNR paradigm was later formulated based on energetic arguments: SN explosions in the Galaxy can easily provide the power needed to sustain the CR population (Morrison 1957; Ginzburg \& Syrovatskii 1964). Assuming that the locally measured CR energy density, $w_{\mathrm{cr}} \sim 1 \mathrm{eV} / \mathrm{cm}^{3}$, is representative of the CR energy density everywhere in the Galaxy, the CR production rate is $\dot{W}_{\mathrm{CR}}=V w_{\mathrm{cr}} / t_{\text {conf }}$ with $t_{\text {conf }}$ and $V$ the CR confinement time and volume, respectively. Using $t_{\text {conf }} \approx 15 \mathrm{Myr}$ (Yanasak et al. 2001) and describing the Galaxy as a cylinder of radius $R_{d} \approx 15 \mathrm{kpc}$ and height $H_{d} \approx 5 \mathrm{kpc}$ (Evoli et al. 2020b; Morlino \& Amato 2020) we can estimate $W_{\mathrm{CR}} \approx 1-3 \times 10^{41} \mathrm{erg} \mathrm{s}^{-1}$.

SN events happen in the Galaxy about every 30 years and typically release $10^{51} \mathrm{erg}$. Thus, the power needed to sustain the galactic CR population turns out to be about $10 \%$ of 
the power provided by SN events. The appeal of the SNR hypothesis was then increased by the formulation of the theory of diffusive shock acceleration (DSA) (Bell 1978; Blandford \& Ostriker 1978): this acceleration process, expected to be active at most shock waves, predicts the spectrum of accelerated particles to be a power law, with an index close to -2 in the case of a strong shock, such as the blast wave of a SN explosion. Such a spectrum perfectly fits what inferred for the sources of Galactic CRs, lending support to the association. An equally remarkable, but much more recent finding, concerns the acceleration efficiency: kinetic simulations of DSA in a regime that is close to representing a SN blast wave have finally become available in the last decade, and they show that for parallel shock waves (magnetic field perpendicular to the shock surface) the acceleration efficiency is 10-20\% (Caprioli \& Spitkovsky 2014a), exactly as required for the SNR-CR paradigm to work.

Testing the SNR paradigm for the origin of CRs has long been one of the top priorities in high-energy astrophysics. Since the early days, photons with energies in the range between $\mathrm{GeVs}$ and TeVs have provided a unique tracer of the CR population far from Earth: the interactions of CRs with the environment are expected, indeed, to produce radiation in this energy range. The process that most directly reveals the presence of hadrons is the decay of neutral pions produced when CR hadrons collide inelastically with ambient gas in the interstellar medium (ISM): this process is commonly referred to as the hadronic production mechanism. As a rule of thumb, an inelastic nuclear collision will produce $\gamma$-rays with $\sim 10 \%$ of the energy of the parent CR, so, for instance, photons of several tens to hundreds of $\mathrm{TeV}$ for $\mathrm{CRs}$ close to PeV. Typically, the spectrum of the hadronic radiation at $\mathrm{TeV}$ mimics the parent $\mathrm{CR}$ spectrum shifted to lower energy by a factor of 20-30 (Kelner, Aharonian \& Bugayov 2006; Kafexhiu et al. 2014). In the same energy range, radiation can originate from leptonic production mechanisms, mainly inverse Compton (IC) scattering of CR electrons off ambient radiation fields, and non-thermal electron bremsstrahlung (Blumenthal \& Gould 1970), which plays an important role in dense gas regions at sub- $\mathrm{GeV}$ to $\mathrm{GeV}$ energies. A recurring difficulty when trying to infer the $\mathrm{CR}$ population from the $\mathrm{GeV}$ and $\mathrm{TeV} \gamma$-ray emission from $\mathrm{SNR}$ s is to break the hadronic-leptonic degeneracy and unveil the dominant emission process: the spectral and morphological features of the emission are crucial to this task.

The $\gamma$-ray spectrum is also affected by the particles' energy losses. For $\gamma$-ray-emitting particles, a number of loss mechanisms that are important at lower energies, such as ionisation and bremsstrahlung, have a negligible impact. The most relevant loss processes affect electrons, whose synchrotron and IC emission must be properly taken into account when trying to disentangle the radiative contribution from leptons and hadrons. Electron synchrotron, particularly efficient in the strong magnetic fields believed to be associated with particle accelerators, has a fundamental role in cooling the CR electron population and thus shape the leptonic $\gamma$-ray spectrum. Therefore, observations of non-thermal radio and X-ray synchrotron emission can provide invaluable constraints on the electron population, and clues to solve the hadronic-leptonic degeneracy.

The SNR paradigm implies that young SNRs accelerate CRs up to the knee and that on average each SNR provides roughly $10^{50} \mathrm{erg}$ in accelerated particles. In order to test whether SNRs are the main contributors to the Galactic CR population, it is thus crucial to assess the energetics in electrons and protons and the spectra of accelerated particles in young SNRs.

Studies of the GeV-to-TeV spectra of the remnants, of their morphology and of the spatial correlation between $\mathrm{TeV} \gamma$-rays and the distribution of ambient gas and of X-ray radiation are the three most powerful instruments to extract the population of accelerated particles in the SNRs. GeV-to-TeV $\gamma$-ray studies of both young SNRs (Drury, Aharonian 
\& Voelk 1994), and dense molecular clouds close to middle-aged SNRs (Aharonian, Drury $\&$ Voelk 1994) were undertaken to assess the role of SNRs in the origin of Galactic CRs, by understanding and disentangling the dynamics of the coexisting and competing processes of acceleration and escape or release of particles in the ISM. A useful repository of high-energy observations of SNRs is the Manitoba Catalogue (http://snrcat.physics. umanitoba.ca/).

Several young shell-type SNRs have been observed at TeV energies. Among these, the best studied are Cas A (Aharonian et al. 2001; Albert et al. 2007a; Ahnen et al. 2017), Tycho (Acciari et al. 2011; Archambault et al. 2017) and RX J1713.7-3946 (Aharonian et al. 2004; Abdalla et al. 2018). We discuss the latter as an example of how the SNR paradigm is tested with radiation from X-rays to $\gamma$-rays.

As an example of multi-wavelength studies concerning middle-aged SNRs showing the so-called pion bump, a clear signature of the presence of relativistic protons, we discuss the case of the brightest GeV source among these: W44.

\subsection{Young SNRs: the case of RX J1713.7-3946}

The SNR RX J1713.7-3946, possibly associated with a star explosion seen by Chinese astronomers in the year AD393 (Pfeffermann \& Aschenbach 1996; Wang, Qu \& Chen 1997), is one of the brightest sources of $\gamma$-rays in the TeV energy range. The sky map of RX J1713.7-3946, obtained with the H.E.S.S. telescope, is shown in the top left panel of figure 1 (Abdalla et al. 2018). The morphology of the TeV shell resembles closely the XMM X-ray shell (Cassam-Chenaï et al. 2004), plotted with blue contours in the top left panel of the figure. The other five panels of figure 1 show the radial profiles at keV and $\mathrm{TeV}$ photon energies from different regions of the shell: in four cases, the TeV SNR extends, in radius, beyond the X-ray emitting shell (Aharonian et al. 2004; Uchiyama et al. 2007; Tanaka et al. 2008; Abdalla et al. 2018). The main shock position and extent are visible in the X-ray data and the $\gamma$-ray emission extending further is either due to accelerated particles escaping the acceleration (shock) region or particles in the shock precursor region.

VHE electrons, up to hundreds of TeV, are accelerated in the shell of RX J1713-3946. These particles emit hard X-rays through synchrotron processes and $\mathrm{TeV}$ radiation through IC scattering off CMB photons very efficiently. A crucial piece of evidence in support of efficient particle acceleration comes from hard X-ray measurements with Suzaku (Uchiyama et al. 2007). Suzaku detected X-ray hot-spots brightening and disappearing within a 1 year timescale. If the X-ray variability is associated with the acceleration and immediate synchrotron cooling of the accelerated electrons, then the magnetic field in the shell must be roughly two orders of magnitude higher than the average magnetic field in the Galaxy, about $100 \mu$ Gauss. Broadband X-ray spectrometric measurements of RXJ1713.7-3946 indicate also that electron acceleration proceeds in the most effective Bohm-diffusion regime. Finally, the presence of strongly amplified magnetic fields lends further support to the idea that not only electrons, but also protons up to $100 \mathrm{TeV}$ are accelerated in the shell, as these amplified magnetic fields would be the result of CR-induced instabilities (Uchiyama et al. 2007).

The high-energy part of the spectrum of RX J1713.7-3946, from X-rays to TeV photons, can be used to infer the population of particles producing this emission. The particle spectrum of the young SNR in the left panel of figure 2 is characterised by a spectral index close to -2 , as predicted by DSA, up to a few $\mathrm{TeV}\left(=10^{12} \mathrm{eV}\right)$. Although the hard spectrum shows that particles are efficiently accelerated in the shell of this young SNR, the cutoff at a few TeV suggests that RX J1713.7-3946 is currently accelerating electrons and protons up to $100 \mathrm{TeV}$ but not up to PeV energies (Abdalla et al. 2018). 
(a)

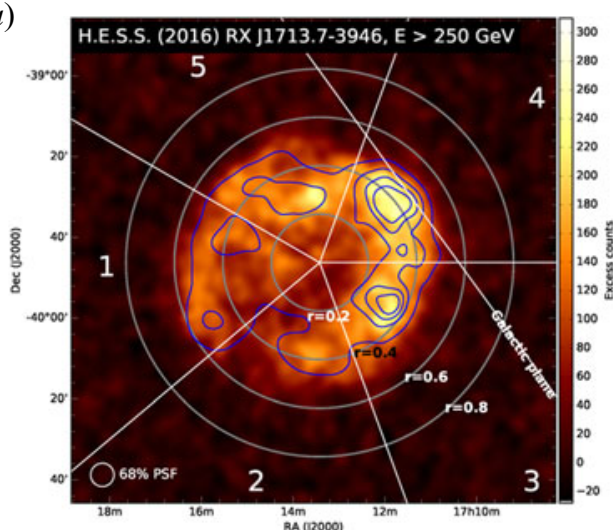

(c)

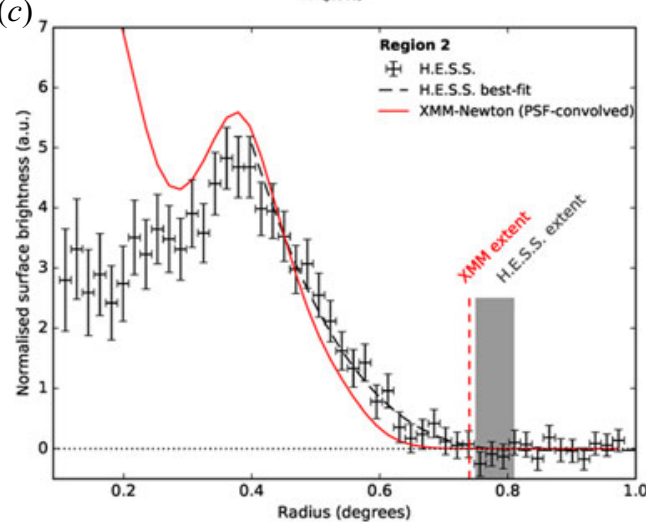

(e)

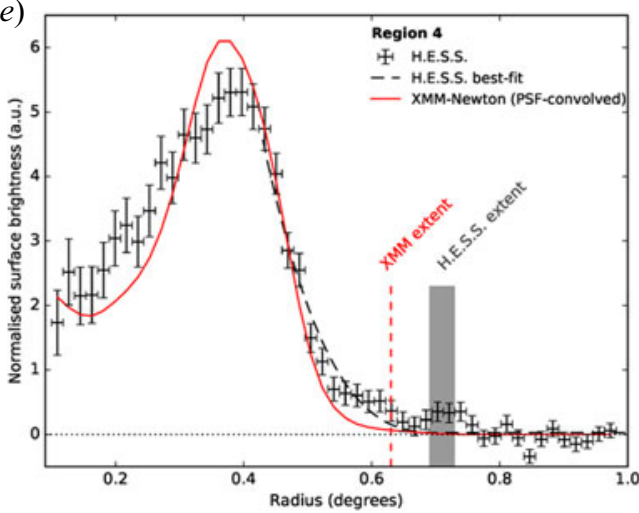

(b)

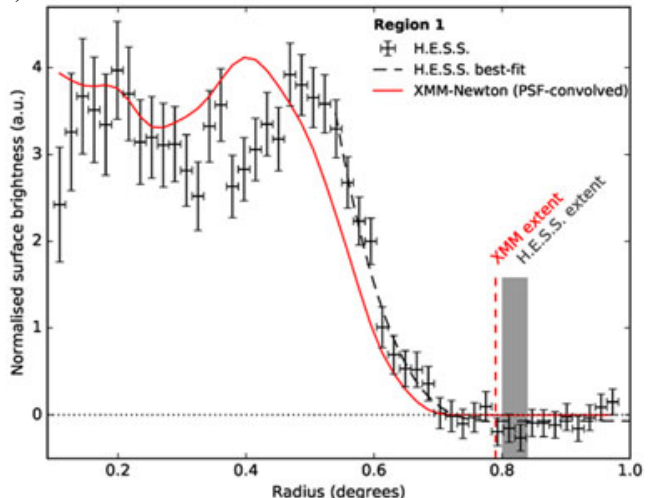

(d)
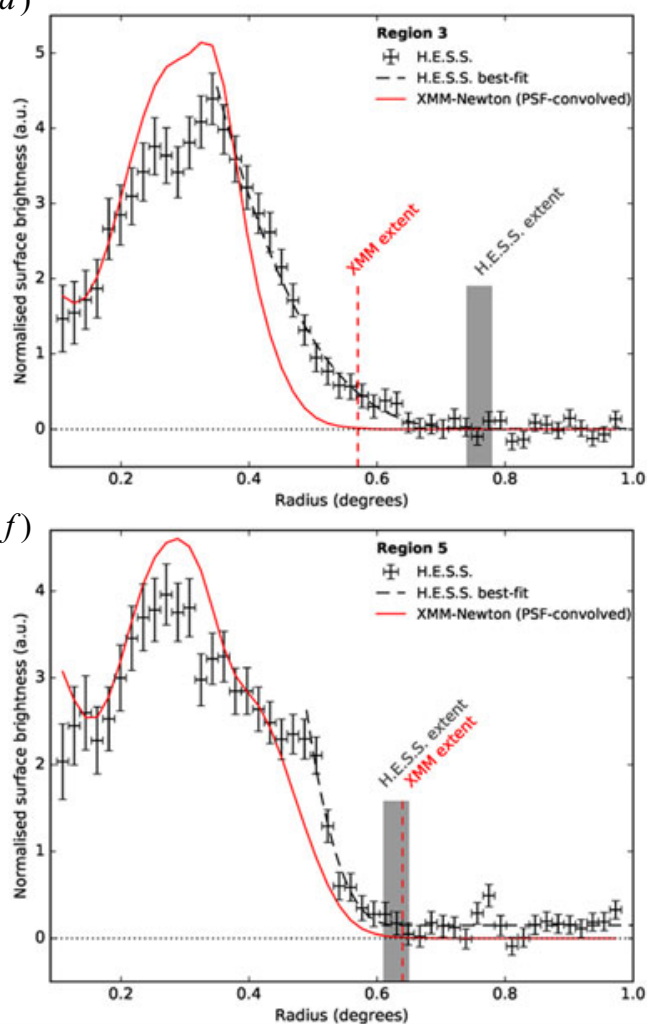

FIGURE 1. Top left panel: H.E.S.S. $\gamma$-ray skymap of RX J1713.7-3946 (Aharonian et al. 2004; Abdalla et al. 2018). In blue the contours of the shell as observed by XMM in X-rays (Cassam-Chenaï et al. 2004) are shown. The morphology of the TeV shell resembles closely the X-ray shell. Top right panel and following: radial profiles of the emission at selected places along the shell from H.E.S.S. and XMM-Newton. In four out of five regions of the shell, the TeV SNR is more extended than the X-ray SNR as a result of possible escape of high energy particles from the shell. The coordinate 'Radius', used for the one-dimensional profiles, refers to the mean radius ' $r$ ' of the shell over which average is performed. 
(a)

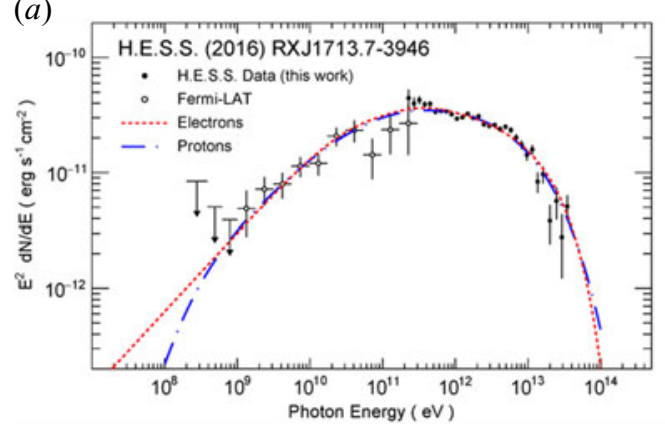

(b)

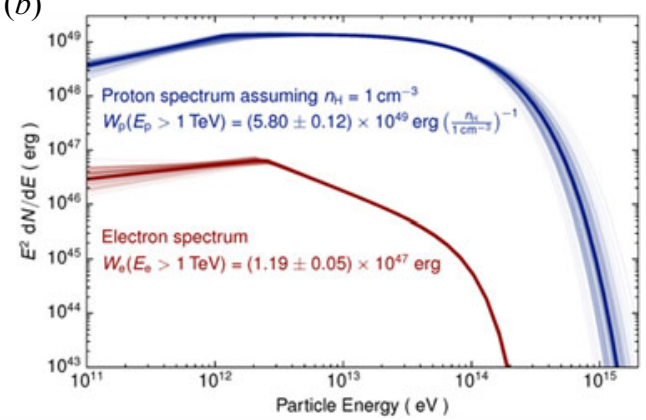

FIgURE 2. (a) The black data points represent the GeV (Fermi-LAT) and TeV (H.E.S.S.) spectral energy distribution of the radiation from the shell of RX J1713.7-3946. The lines represent the multi-wavelength spectra predicted by NAIMA (Zabalza 2015), assuming either a leptonic or hadronic origin of the emission. $(b)$ The spectral energy distributions of the electrons and protons producing the leptonic and hadronic emissions shown on the left panel.

As mentioned, protons are likely accelerated in the SN shell through the same mechanism accelerating electrons, but it is not clear what fraction of the energy input by the SN explosion goes into the acceleration of electrons and what fraction goes into the acceleration of protons. Both protons or electrons can be, in fact, responsible for the $\mathrm{TeV}$ emission from RX J1713.7-3946 (leptonic-hadronic degeneracy, see e.g. Morlino, Amato \& Blasi 2009). If electrons are responsible for the emission from the shell, by IC scattering of the ambient radiation fields, one speaks of the leptonic scenario. On the other hand, if the emission is produced by protons colliding with the ambient gas, one speaks of the hadronic scenario. In figure 2 the measurements of the shell emission carried out by Fermi LAT (Abdo et al. 2011), HESS (Abdalla et al. 2018) and Suzaku (Tanaka et al. 2008) are compared with leptonic (left panel) and hadronic (right panel) model predictions by Zabalza (2015). The total energetics in accelerated electrons and protons in the relevant leptonic and hadronic models of $\gamma$-rays can be estimated by assuming a distance to the source of about $1 \mathrm{kpc}$ (Pfeffermann \& Aschenbach 1996; Wang et al. 1997). The required budget in electrons is determined only by the reported $\gamma$-ray fluxes, if the target radiation is the CMB: one finds $W_{\mathrm{e}} \simeq 1.2 \times 10^{47} \mathrm{erg}$. On the other hand, the total energy budget of protons in hadronic models depends on the highly uncertain ambient gas density: $W_{\mathrm{p}} \simeq 5 \times 10^{49}\left(n_{H} / 1 \mathrm{~cm}^{-3}\right)^{-1} \mathrm{erg}$. The leptonic model in the left panel corresponds to the electron IC scattering off $\mathrm{CMB}$ photons and an infrared radiation field with energy density $0.415 \mathrm{eV} \mathrm{cm}^{-3}$ and temperature $T=26.5 \mathrm{~K}$.

The leptonic model requires a break in the electron spectrum at $2.5 \mathrm{TeV}$ with the index of the electron energy distribution changing from $\Gamma=1.7$ to $\Gamma=3$ beyond the break. If, due to synchrotron cooling, this spectral break implies a magnetic field of about $140 \mu \mathrm{G}$, which is much larger than what the $\mathrm{X}$-ray flux allows within the same scenario, $B \approx 15 \mu \mathrm{G}$. Similar difficulties arise if one tries to explain the break as a result of IC cooling, in which case a photon field of energy density of $140 \mathrm{eV} \mathrm{cm}^{-3}$ would be required, about 100 times larger than the average galactic value.

If the emission is hadronic in origin, a spectrum of protons with index $\Gamma=1.5$, steepening to $\Gamma=1.9$ at about $1 \mathrm{TeV}$, and with an exponential cutoff at $79 \mathrm{TeV}$ is required. The break in the proton population spectrum can be explained if the hadronic emission is produced mostly in dense clumpy regions. In fact, low-energy protons can be efficiently excluded from dense gas region during the timescale of $\sim 1000$ years since 
the SNR explosion. The exclusion of low-energy CRs would also explain the hard $\gamma$-rays detected by the Fermi LAT telescope (Fukui et al. 2012; Inoue et al. 2012; Gabici \& Aharonian 2014), and the lack of thermal X-ray emission from the shell. The latter has traditionally been one of the strongest arguments against a possible hadronic origin of the emission from the shell of RXJ1713.7-3946, because it implies an ambient gas density as low as $0.1 \mathrm{~cm}^{-3}$ (Ellison et al. 2010; Fukui et al. 2012; Gabici \& Aharonian 2014). Finally, hadronic $\gamma$-ray production in gas condensations results in a narrow angular distribution of the radiation, which is beyond the reach of the current generation of Imaging Air Cherenkov Telescopes (IACTs), but could be tested with the upcoming Cherenkov Telescope Array (CTA), which will have an angular resolution of $\sim 1-2$ arcmin (Zirakashvili \& Aharonian 2010; Ambrogi, Celli \& Aharonian 2018).

The conclusion one reaches, after investigating the spectrum and morphology of RX J1713.7-3946 as currently known, is that neither the hadronic nor the leptonic scenario is fully satisfactory. Each emission mechanism has strengths and weaknesses when compared with observations, suggesting that the ambient conditions might differ in different parts of the remnant, making one or the other process locally dominant. Although for some specific SNRs one of the two scenarios might indeed be favoured (see e.g. Funk 2015), the general conclusion is that no known SNR has proved to accelerate particles beyond $100 \mathrm{TeV}$.

\subsection{Molecular clouds close to middle-aged SNRs: the case of W44}

Molecular clouds are regions of the Galaxy, typically a few tens of parsecs in radius, where the density of cold molecular gas is often orders of magnitude higher than elsewhere in the diffuse ISM. Stars are believed to be born in these clouds. Radio observations of the rotational $1 \rightarrow 0$ line emission of carbon monoxide are mainly used to trace the distribution of molecular gas (Heyer \& Dame 2015). The cloud Galactocentric distance is usually estimated using a kinematical distance method, through each the radial velocity of the cloud is related to the rotation velocity of the Galaxy. Cross-calibrations with the distance of spiral arms or known objects with precise parallax determination are also carried out (Roman-Duval et al. 2009; Reid et al. 2014).

Giant molecular clouds, which are typically 5 to 200 parsecs in diameter and have masses of 10000 to 10 million Solar masses (Murray 2011), are excellent laboratories for CR physics. In these clouds, the hadronic channel of $\gamma$-ray production is enhanced by the high target density, and easily dominates over the leptonic production mechanism. In contrast to the warmer atomic gas phase, which is homogeneously distributed in the Galaxy, one or a few giant molecular clouds are essentially the dominant contributions to the gas column density along a given direction in the sky. The emission enhancement associated with the clouds makes it possible to precisely locate along a given line of sight where the CR population produces the emission. Molecular clouds are thus used to perform a sort of tomography, and obtain a three-dimensional view of the CR distribution in the Galaxy (Issa \& Wolfendale 1981; Aharonian \& Atoyan 1996; Aharonian 2001; Casanova et al. 2010a; Aharonian et al. 2020; Baghmanyan et al. 2020).

The plasma conditions in molecular clouds are generally different from those in the diffuse ISM. In addition to the gas density, the magnetic field energy density and turbulence level are also enhanced (see e.g.Crutcher (2012) for a comprehensive review). This will lead to a suppression of the diffusion coefficient and effective exclusion of lower-energy CRs from the cloud (Gabici, Aharonian \& Blasi 2007). If CRs can penetrate clouds, the $\gamma$-ray emission from $\pi^{0}$-decay depends only upon the total mass of the cloud, $M_{c l}$, its distance from the Earth, $d$, and the CR flux within the cloud, $\Phi_{\mathrm{CR}}$. The latter is thus determined as $\Phi_{\mathrm{CR}} \propto \Phi_{\gamma} d^{2} / M_{c l}$, where $\Phi_{\gamma}$ is the $\gamma$-ray flux from the cloud. 

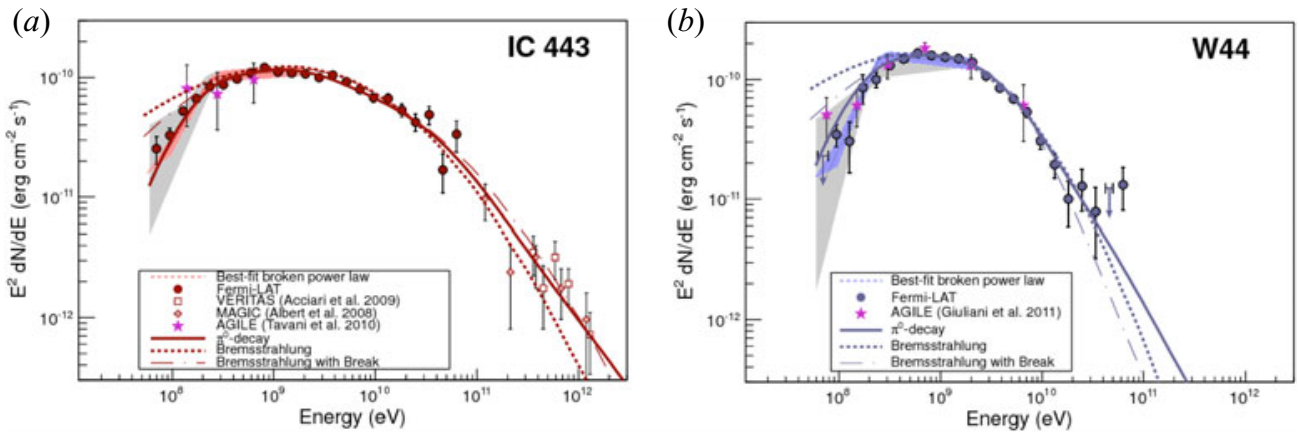

FIGURE 3. Gamma-ray spectra of IC443 $(a)$ and W44 $(b)$ as measured with the Fermi LAT (Abdo et al. 2010a), in blue, and AGILE (Giuliani et al. 2011), in magenta. The pion bump feature at about $1 \mathrm{GeV}$ is evident in the spectra of both SNRs. For IC433 the data points of MAGIC (Albert et al. 2007b) and VERITAS (Acciari et al. 2009) are also shown. Colour-shaded areas denote the best-fit broadband smooth broken power law (60 MeV to $2 \mathrm{GeV}$ ); grey-shaded bands show Fermi LAT systematic errors below $2 \mathrm{GeV}$. The data points at the highest energy $(4 \times$ $10^{10}-1 \times 10^{11} \mathrm{eV}$ ) suggest a hardening of the spectrum, which might be produced by runaway CRs illuminating a molecular cloud (MC) located in front of the shells.

Lower-energy CRs can be effectively excluded from penetrating clouds, which results in peculiar features in the $\gamma$-ray spectrum from clouds, such as hardenings with respect to the average interstellar spectrum (Gabici et al. 2007).

The role of molecular clouds in testing the SNR paradigm is made particularly crucial by the time evolution of particle acceleration in SNRs. Within a DSA scenario, SNRs accelerate the highest-energy CRs (in principle, up to a few $\mathrm{PeV}$, but see $\S 3$ ) at the transition between the free expansion and the Sedov phase, which typically happens a few tens to a few hundred years after the SN explosion, depending on the explosion type and properties of the surrounding medium. During the Sedov phase, the SN shock slows down and the magnetic field intensity decreases, so that the most energetic particles cannot be confined any longer and are free to escape. In practice, a SNR can accelerate the highest-energy particles only for a short time. This fact, coupled to the low rate of PeV particles accelerating events (see § 3) makes the chances of observing a SNR when it still acts as a PeVatron very low. The runaway CRs can illuminate molecular clouds located close to the SNRs and this enhanced $\gamma$-ray emission can thus provide crucial insights on the parental population of runaway CRs, which would otherwise escape the SNR without leaving a footprint (Montmerle \& Cesarsky 1979; Issa \& Wolfendale 1981; Aharonian \& Atoyan 1996; Aharonian 2001; Gabici et al. 2007). The emission produced by these runaway $\mathrm{CRs}$ is essential to trace back acceleration up to $\mathrm{PeV}$ energies.

Gamma-rays in association with dense molecular clouds located close to SNRs have been detected both at $\mathrm{GeV}$ and $\mathrm{TeV}$ energies. Depending on the location of massive clouds, on the acceleration history and on the timescales of the particle escape into the ISM (which depend, in turn, on the diffusion coefficient), a broad variety of energy distributions of $\gamma$-rays is produced, from very hard (much harder than the spectrum of the SNR itself) to very steep spectra (Aharonian \& Atoyan 1996; Gabici, Aharonian \& Casanova 2009; Casanova et al. 2010b).

In figure 3 we show the spectrum of two bright middle-aged SNRs, IC443 and W44. W44 is a 20 000-year-old SNR located on the Galactic Plane at a distance of roughly $3 \mathrm{kpc}$ from the Sun. W44, which is the brightest middle-aged SNR at GeV energies, is a perfect 
laboratory to test the coexisting processes of acceleration and escape of CRs from SNRs. Owing to the slow shock speed, high-energy particles are expected to have already escaped from this source. A population of protons with spectral index close to -2.3 and a cutoff at about $80 \mathrm{GeV}$ is likely responsible for the $\gamma$-ray emission from the remnant measured by AGILE and Fermi-LAT (Abdo et al. 2010a; Giuliani et al. 2011). The presence of such a low-energy cutoff might be the effect of the escape of the highest-energy CRs. Indeed, the remnant is thought to be currently a rather poor accelerator and most of its $\gamma$-ray emission can be interpreted as due to re-acceleration of ambient CRs, rather than to acceleration of fresh particles (Uchiyama et al. 2010; Cardillo et al. 2016).

Owing to their short lives, SNRs are often found within the giant molecular gas complexes where they were born as massive stars. W44 resides within a giant gas complex of $10^{6}$ Solar masses, homogeneously distributed over an extended region surrounding the remnant (Dame, Hartmann \& Thaddeus 2001). North west and south east of the remnant, Uchiyama et al. (2012) discovered two bright sources, which the authors associated to runaway CRs colliding with the dense gas clouds. Peron et al. (2020) re-analysed both the Fermi-LAT data and the gas data from the clouds around W44 and noted that, despite the gas is distributed homogeneously, the $\mathrm{GeV}$ emissivity is enhanced only in the two regions which can be associated to regions of enhanced CR density, CR clouds, rather than gas clouds as previously thought. This phenomenon suggests the existence of a preferential path for CR escape, likely linked to the magnetic field structure in the vicinity of this source.

\section{Recent developments on CR acceleration in SNRs}

Despite all the recent observational developments, providing several hints of efficient CR acceleration in SNRs, a number of unsettled questions remain and challenge the association. In particular, one of the strongest arguments to look for alternative particle accelerators has to do with the difficulties SNRs have at reaching PeV energies. This aspect of the CR-SNR connection has long appeared as the most delicate.

As already mentioned, CR acceleration in SNRs is thought to be well described within the framework of DSA. Acceleration mechanisms alternative to DSA have also been proposed and studied (see e.g. Lazarian et al. 2020). In this article, however, we focus on DSA, which is still the best studied and the most promising, in this context, to reach the highest energies. The idea at the basis of the theory is that particles gain energy each time they cross the shock thanks to the discontinuity of the fluid velocity field at the shock, that leaves an unscreened electric field. The energy gain of the particle is a constant fraction $\left(v_{\mathrm{sh}} / c\right.$ with $v_{\mathrm{sh}}$ the shock velocity) of its energy before the crossing. The particle moves diffusively between crossings, scattering on low-frequency magnetic turbulence and continuously changing its pitch angle. Reaching high energies requires a large number of crossings, which have to occur in a time shorter than the minimum between the duration of the system $t_{\text {life }}$ and the time scale over which energy losses become important $t_{\text {loss. }}$. In the case of protons losses are negligible and the energy is limited by the time for which the SNR is active as an accelerator, $t_{\text {life }}<10^{4} \mathrm{yr}$. Then $t_{\text {life }}$ must be compared with the acceleration time, $t_{\text {acc }}$, which depends on how quickly the particle is able to go back to the shock after each crossing: we can estimate $t_{\text {acc }}(E) \approx D(E) / v_{\mathrm{sh}}^{2}$, where $D(E)$ is the particle diffusion coefficient, typically an increasing function of the particle energy $E$. For relatively low turbulence levels one can estimate $D(E)$ in quasi-linear theory, writing it as (see e.g. Amato 2014)

$$
D(E) \approx \frac{v(E) r_{L}(E)}{3} \frac{1}{k W(k)},
$$


where $v(E)$ is the particle velocity, $r_{L}(E)$ its Larmor radius, and $W(k)$ is the spectrum of magnetic fluctuations causing the diffusion, with

$$
\int_{1 / L}^{\infty} W\left(k^{\prime}\right) \mathrm{d} k^{\prime}=\frac{\delta B^{2}}{B_{0}^{2}},
$$

and $k$ the wave number resonant with the particle of energy $E$, namely $k=1 / r_{L}(E)$.

Assuming that the scattering is due to turbulence that is injected by SNRs on a typical scale $L \approx 100$ pc with $\delta B / B_{0} \approx 1$, and then develops a Kolmogorov-type spectrum $W(k) \propto k^{-5 / 3}$, from (3.1) and (3.2), ${ }^{1}$ one can estimate the diffusion coefficient as $D(E)=$ $7 \times 10^{27}(E / G e V)^{1 / 3} \mathrm{~cm}^{2} \mathrm{~s}^{-1}$, which, as we discuss in the following, is not very different from what $\mathrm{CR}$ observations indicate $\left(D(1 \mathrm{GeV}) \approx 10^{28} \mathrm{~cm}^{2} \mathrm{~s}^{-1}\right)$. If this estimate of the diffusion coefficient were appropriate to describe particle transport in the vicinity of a SNR shock, the maximum achievable energy in these systems would only be $E_{\max } \sim$ few $\mathrm{GeV}$, and could only improve to $\sim 100 \mathrm{TeV}$ if particle transport were described by Bohm diffusion, namely $\delta B / B_{0} \approx 1$ at all relevant scales (Lagage \& Cesarsky 1983). It is then clear that in order to reach PeV energies, not only is efficient (Bohm-like) scattering needed, but so are largely amplified magnetic fields.

As already mentioned, this is exactly what X-ray observations of young SNRs were found to show: aside from the short variability time scales already discussed in $\S 2.1$, $\mathrm{X}$-ray synchrotron emission is seen to be confined to rims of thickness $\Delta \sim$ few $\times 0.01$ pc (Vink 2012). The extremely small value of $\Delta$ can be interpreted either as a result of radiative losses, that kill the electron population with increasing distance from the shock, or as a result of magnetic field damping (Rettig \& Pohl 2012). If we interpret the thickness of the rims as the distance travelled by the emitting electrons in a synchrotron loss time, we can write $\Delta \approx \sqrt{D(E) t_{\text {sync }}(E)}=0.04$ pc $B_{-4}^{-3 / 2}$ with $B_{-4}$ the magnetic field downstream of the shock in units of $10^{-4} \mathrm{G}$. It is clear then that a magnetic field amplified by a factor of $\sim 100$ with respect to the interstellar value $\left(B_{\text {ISM }} \approx 3 \mu \mathrm{G}\right)$ is implied in the rims, if they are to be interpreted as the result of radiative losses. On the other hand, interpreting $\Delta$ as a result of magnetic damping requires a value of the magnetic field which is still in the same range estimated previously (Pohl, Yan \& Lazarian 2005), and hence largely amplified.

There are several mechanisms by which a magnetic field can be amplified at a shock (Bykov, Ellison \& Renaud 2012): some involve fluid instabilities (Giacalone \& Jokipii 2007; Ohira 2016), some involve magnetohydrodynamic (MHD) instabilities and CR-related effects (Beresnyak, Jones \& Lazarian 2009), some others involve CR-induced instabilities, either of resonant type ( see Amato \& Blasi 2006 and references therein) or of non-resonant type (Bell 2004; Reville et al. 2008; Amato \& Blasi 2009). Except for purely fluid instabilities (Giacalone \& Jokipii 2007), all other classes require efficient CR acceleration, and in this sense the detection of amplified magnetic fields can be considered as indirect evidence of efficient CR acceleration in SNRs. However, the different mechanisms proposed are not equivalent in terms of the spectrum of magnetic fluctuations they produce and, hence, in terms of consequences on particle acceleration. Particle scattering is only efficient with resonant waves. Hence, MFA will increase scattering efficiency and lead to high maximum energies of the accelerated particles only if there is enough power on scales comparable with the particle Larmor radii. The most

\footnotetext{
${ }^{1}$ One may wonder about the appropriateness of using quasi-linear theory for turbulence levels as large as $\delta B / B_{0} \approx 1$ : although this is not fully justified on theoretical ground, numerical simulations find it to provide a decent description of wave-particle interactions (see e.g.Holcomb \& Spitkovsky 2019)
} 
promising MFA mechanism in this sense turns out to be the non-resonant CR streaming instability (Bell's instability).

The super-Alfvénic streaming of energetic particles has long been known to induce the growth of magnetic fluctuations at wavelengths that are resonant with the gyroradii of the exciting particles (Skilling 1975): this is the so-called resonant streaming instability. While creating fluctuations on the right scales, this instability can only lead to MFA up to a level $\delta B / B_{0} \lesssim 1$, and hence it is not powerful enough to explain the field strength deduced in SNRs, nor to guarantee particle acceleration up to the knee. In more recent times, however, it has been recognised that a more powerful instability arises if the CR current is large enough to twist the ambient magnetic field on the scale of the Larmor radius $\left(r_{L, 0}\right)$ of the particles that carry the current $\left(J_{\mathrm{CR}}>(c / 4 \pi)\left(B_{0} / r_{L, 0}\right)\right)$ or, equivalently, if the CR energy density, $U_{\mathrm{CR}}$, is larger than $c / v_{D}$ times the magnetic energy density (Bell 2004):

$$
U_{\mathrm{CR}}>\frac{c}{v_{D}} \frac{B_{0}^{2}}{4 \pi},
$$

where $v_{D}$ is the bulk velocity of CRs. If this condition is satisfied, the magnetic field grows very rapidly. The basic physical process can be described as follows: the CR current induces a compensating return current in the ISM plasma; the force $\vec{J}_{\text {ret }} \wedge \vec{B}$ induces transverse plasma motion; the current associated with this motion acts as a source of magnetic field; the result is that the magnetic field lines associated with right-hand polarised waves are stretched and for these modes the field is amplified.

In the vicinity of a shock that is accelerating particles, (3.3) can be turned into a condition for the acceleration efficiency $\xi_{\mathrm{CR}}$ :

$$
\xi_{\mathrm{CR}}>\frac{c B_{0}^{2}}{8 \pi \rho_{\mathrm{ISM}} v_{\mathrm{sh}}^{3}} .
$$

Here $\xi_{\mathrm{CR}}$ is the fraction of incoming flow energy $\left(\rho_{\mathrm{ISM}} v_{\mathrm{sh}}^{2} / 2\right)$ that is converted into accelerated particles, $\rho_{\mathrm{ISM}}$ is the mass density of the ISM plasma and $v_{\mathrm{sh}}$ is the velocity of the blast wave, also coincident with the CR bulk velocity. Equation (3.4) makes it clear that the possibility for the non-resonant streaming instability to operate strongly depends on the shock velocity: detailed calculations show that it is indeed a viable MFA mechanism in the vicinity of the fast shocks of young SNRs (Amato \& Blasi 2009), but then stops working early during the Sedov-Taylor phase of expansion of the blast wave, typically after a few hundred to a few thousand years, depending on the ambient medium density and magnetic field strength.

The growth initially occurs on very small scales $\left(k \approx(4 \pi / c)\left(J_{\mathrm{CR}} / B_{0} \gg r_{\mathrm{L}, 0}\right.\right.$, but quickly the power moves to larger and larger scales (Riquelme \& Spitkovsky 2009), possibly due to some mean-field dynamo process (Bykov, Osipov \& Ellison 2011; Rogachevskii et al. 2012), and numerical simulations show that the final outcome of the instability, when it develops at a shock, is a spectrum of fluctuations with $W(k) \propto k^{-1}$, leading to Bohm diffusion of the particles (Caprioli \& Spitkovsky 2014c). When saturation is reached, the magnetic energy density is a substantial fraction of the CR energy density (Caprioli \& Spitkovsky 2014b).

The level of MFA that the non-resonant streaming instability can provide is in the correct range to explain the magnetic field strength inferred in SNRs (Schure et al. 2012), and as we just mentioned the associated scattering is efficient (Caprioli \& Spitkovsky 2014c), and yet recent studies have cast doubt on the fact that these sources might be able to 
accelerate particles up to PeV energies (Cardillo, Amato \& Blasi 2015). In fact, assuming that MFA is primarily due to the streaming of particles that leave the acceleration region, it is possible to build a description of the shock as a self-regulating system: when the level of turbulence in the upstream is low, a large fraction of particles can escape from the shock; when this happens, however, the large current in the upstream causes the growth of turbulence and escape is reduced; if the fraction of escaping particles becomes too small, then the turbulence level is reduced again favouring escape. The self-regulation mechanism qualitatively illustrated previously translates into a quantitative prescription for the maximum energy of shock-accelerated particles as a function of the system parameters. The current of escaping particles, which is what determines the level of MFA, is, in turn, determined by the shock velocity and the spectrum of accelerated particles, including its total energy content (which determines the amount of energy available), its slope and the maximum particle energy (which determine the current, once the energy content is fixed). When writing the equation describing $E_{\max }$ as a function of time during the expansion of a $\mathrm{SN}$ blast wave, one finds that $E_{\max }$ is an ever-decreasing function of time during the SNR evolution, so that the relevant value of $E_{\max }$ is that reached at the beginning of the Sedov-Taylor phase, namely the highest possible after a sufficient amount of mass has been processed by the blast wave. It is clear then that a higher maximum energy will be achieved in systems that enter the Sedov-Taylor phase earlier in time after the explosion. This occurs for type II explosions expanding in the dense and slow wind of a progenitor red super-giant star. In this case, assuming a $\propto E^{-2}$ spectrum, the maximum achievable energy reads

$$
E_{\max } \approx 0.5\left(\frac{\xi_{\mathrm{CR}}}{0.1}\right)\left(\frac{E_{\mathrm{SN}}}{10^{51} \mathrm{erg}}\right)\left(\frac{M_{\mathrm{ej}}}{M_{\odot}}\right)^{-1}\left(\frac{\dot{M}}{10^{-5} M_{\odot} / \mathrm{yr}}\right)^{1 / 2}\left(\frac{v_{w}}{10 \mathrm{~km} \mathrm{~s}^{-1}}\right)^{-1 / 2} \mathrm{PeV}
$$

where $\xi_{\mathrm{CR}}$ is the $\mathrm{CR}$ acceleration efficiency, $E_{\mathrm{SN}}$ and $M_{\mathrm{ej}}$ are the energy released and the mass of material ejected in the SN explosion, and $\dot{M}$ and $v_{w}$ are the mass loss rate and speed of the progenitor's wind. If one takes into account that the mass of the ejecta in a type II SN explosion is more likely around $10 M_{\odot}$, it is immediately clear that reaching the knee is very challenging in this framework. One is forced to invoke extremely energetic events, or extreme acceleration efficiency, or finally extreme properties of the progenitor. In all cases these must be rare events. In fact, the product of $\xi_{\mathrm{CR}}, E_{\mathrm{SN}}$ and the frequency in the Galaxy of the PeV producing SN explosions are constrained by the overall flux of CRs measured flux at Earth. When all of this is taken into account, one determines an event rate that cannot exceed a few in $10^{4}$ yr (Cristofari, Blasi \& Amato 2020).

In reality the possibility for SNRs to reach the knee becomes even more challenging when taking into account the fact that the accelerated CR spectrum is likely $\propto E^{-p}$ with $p>2$. Such a spectrum corresponds to a smaller current for a given $E_{\max }$ and total energy in accelerated particles, hence requiring even more extreme parameters to reach $E_{\max } \approx$ $\mathrm{PeV}$ (e.g.Cardillo et al. 2015). On the other hand a spectrum steeper than $E^{-2}$ is exactly what $\gamma$-ray observations of the majority of young SNRs require and what recent progress on propagation also seems to require (Evoli, Aloisio \& Blasi 2019): the CR spectrum released in the ISM will be steeper than $E^{-2}$ only if the spectrum in the source is itself steeper than $E^{-2}$ (Schure \& Bell 2014; Cardillo et al. 2015) and a steep injection spectrum of CRs is exactly what the most recent CR data seem to suggest, as we discuss in $\S 4$. 


\section{CR transport in the Galaxy}

As mentioned in $\S 1$ the last decade has also been rich with observational progress providing interesting constraints on the properties of CR transport throughout the Galaxy. In this section we review the main findings and try to put them in a coherent theoretical framework.

The first important discovery was that of a hardening in the spectrum of protons and $\mathrm{He}$ nuclei (Adriani et al. 2011; Aguilar et al. 2015a,b) and also, though with somewhat lower statistics, of heavier nuclei (Ahn et al. 2010). Within the framework of diffusive transport, the detection of a break in the spectrum of CRs can be interpreted as a signature of a change either in the injection spectrum or in the diffusion coefficient. During propagation through the Galaxy, CRs primarily loose energy due to adiabatic expansion and ionisation. In addition, particles of a given species can be also lost due to spallation or decay. However, if one focuses on stable primary CRs, with energy above a few tens of GeV, energy losses during propagation can be neglected and a very simple expression for the diffuse steady-state spectrum in the Galaxy can be found. If we assume that CRs are injected in the Galaxy at a constant rate

$$
Q_{\mathrm{p}}(E) \propto E^{-\gamma_{\mathrm{inj}}},
$$

and that particles then propagate diffusively with an average diffusion coefficient

$$
D(E) \propto E^{\delta},
$$

the steady-state spectrum of stable CR nuclei in the Galaxy will be given by the product between injection and confinement time, $Q(E) \times \tau_{\text {esc }}$. This will read, for primary nuclei,

$$
N_{\mathrm{p}}(E) \propto Q(E) \frac{H^{2}}{D(E)} \propto E^{-\gamma_{\mathrm{inj}}-\delta},
$$

where the confinement time has been taken to be $\tau_{\text {esc }}=H^{2} / D(E)$, with $H$ the size of the magnetised halo in which CRs are confined (see e.g. Amato \& Blasi 2018 for a more refined description). It is then clear then that the detected hardening implies a change in $\gamma_{\text {inj }}$ or $\delta$. Several models have been proposed invoking either of the two (Tomassetti 2012, Thoudam \& Hörandel 2012, and references therein). A possibility is that this feature is signalling the importance of non-linear effects in CR propagation. An early suggestion (Blasi, Amato \& Serpico 2012) was that the break could point to the transition between scattering in self-generated and external turbulence. It had indeed been suggested since the 1970s (Wentzel 1974; Cesarsky 1980) that at scales comparable with the Larmor radius of $\mathrm{GeV}$ particles, CRs could be an important source of turbulence in the galaxy through the resonant streaming instability. At larger scales, on the other hand, CRs become too few, given their steep spectrum, and the main source of scattering would become the large-scale turbulence present in the Galaxy. The latter is usually assumed to be injected by SNRs on a scale of order tens to $100 \mathrm{pc}$ and then cascade to smaller scales developing a Kolmogorov-type spectrum $k^{-5 / 3}$. Such a spectrum translates in a diffusion coefficient with $\delta \approx 1 / 3$, flatter than the low-energy value $\delta \approx 0.7$ that is appropriate to describe CR self-generated turbulence. A back of the envelope calculation places the transition between self-generated and external turbulence at a CR rigidity in the range 200-300 GV (Blasi et al. 2012), tantalisingly close to the value of $336 \mathrm{GV}$ at which AMS-02 detects the hardening in the proton spectrum.

This explanation of the hardening as due to a change in the properties of galactic transport entails a clear prediction for the spectrum of secondary CR nuclei. These are 
injected in the Galaxy as a result of spallation of primaries. If we approximate the spallation cross-section as independent of energy, their injection will be

$$
Q_{\mathrm{sec}}(E) \propto N_{p}(E) \sigma_{\mathrm{sp}} c n_{\mathrm{ISM}} \propto E^{-\gamma_{\mathrm{inj}}-\delta},
$$

where $n_{\mathrm{ISM}}$ is the target gas density. Their equilibrium spectrum in the Galaxy will then read

$$
N_{\text {sec }}(E) \propto Q_{\text {sec }}(E) \tau_{\text {esc }} \propto E^{-\gamma_{\text {inj }}-2 \delta},
$$

which implies that any hardening $\Delta$ of the spectrum of primaries due to a change in the slope of the diffusion coefficient will reflect in a hardening of the spectrum of secondaries equal to $2 \Delta$ : this expectation is perfectly consistent with the analysis of secondaries performed by AMS-02 (Aguilar et al. 2018).

Within this interpretation of the observed hardenings, the transport of CRs through the Galaxy becomes a complex non-linear problem, where the diffusion coefficient and the spectra of all nuclei need to be determined self-consistently. Once this complex problem is solved, however, and all available AMS-02 data are reproduced, the low-energy Voyager data (Stone et al. 2013) are automatically reproduced (Aloisio, Blasi \& Serpico 2015). One important result that comes out of this analysis is directly related to the boron-over-carbon (B/C) measurements of AMS-02 at high energies (Aguilar et al. 2016b). The $\mathrm{B} / \mathrm{C}$ ratio has traditionally been considered the primary indicator of $\mathrm{CR}$ transport: $\mathrm{B}$ is the most abundant stable secondary and its mostly produced by the spallation of $\mathrm{C}$, though the contribution by $\mathrm{N}$ and $\mathrm{O}$ is not negligible. In practice, the $\mathrm{B} / \mathrm{C}$ ratio provides a direct measurement of the grammage (mass per unit surface, or mass density integrated along the path length) encountered by CRs during their propagation from their sources to Earth. If one compares (4.5) and (4.3), for the spectrum of secondary and primary nuclei, respectively, one immediately sees that the ratio between the two is expected to scale with energy exactly as the diffusion coefficient. In fact this ratio, as measured by AMS-02 (Aguilar et al. 2016b), well agrees with a high-energy slope of the diffusion coefficient $\delta \approx 0.4$ (Aloisio et al. 2015; Evoli et al. 2019), but an additional, energy independent contribution seems to be required to well reproduce not only the highest-, but also the lowest-energy (Cummings et al. 2016) available data. The additional grammage needed, $X_{s} \approx 0.15 \mathrm{~g} \mathrm{~cm}^{-2}$, is independent of energy and of the order of what particles can accumulate within a SNR, or any source with an ambient density of order $1 \mathrm{~cm}^{-3}$ and a duration $\approx 10^{4} \mathrm{yr}$, during acceleration (Aloisio et al. 2015; Bresci et al. 2019). Within this modelling, namely if hardenings are interpreted as a result of turbulence self-generation at low energies, the contribution $X_{s}$ turns out to be fundamental also for explaining another recent surprise found in CR data, namely the spectrum of anti-protons, which we discuss in $\$ 6$.

Before concluding this section we would like to remark on the most important consequence of a scenario in which $\mathrm{CR}$ propagation at high energy is described by a diffusion coefficient $\delta \approx 0.4$ : CRs must be injected in the galaxy with a spectrum $E^{-\gamma_{\text {inj }}}$ with $\gamma_{\text {inj }} \approx 2.3$. This combination of injection and propagation parameters and, in particular, the relatively weak energy dependence of the diffusion coefficient, helps to explain the low level of anisotropy detected at TeV energies, as shown by Blasi \& Amato $(2012 a, b)$ and Sveshnikova, Strelnikova \& Ptuskin (2013). On the other hand, as discussed in $\S 3$, such a steep spectrum makes it very difficult for SNRs to be the primary sources of PeV CRs.

Within a DSA scenario, SNRs accelerate the highest-energy CRs (up to at least a few $\mathrm{PeV}$ ) at the transition between the free expansion and the Sedov-Taylor phase, which typically happens a few hundred years after the SN explosion. During the Sedov-Taylor 
phase the SN shock slows down and the magnetic field intensity decreases, so that the shock cannot confine any longer the most energetic particles, which escape the SNR. This means that a SNR can act as a PeVatron for a relatively short time. Considering the rate of SN explosions in the entire Galaxy (about three per century), the chances of observing a SNR when it is still a PeVatron are thus very low, and any proof of emission from PeV CRs even from young SNRs is challenging to find.

\section{Alternative CR sources}

Although $\gamma$-ray observations have proven that SNRs are efficient accelerators of CR electrons, and possibly protons, up to $100 \mathrm{TeV}$, the hypothesis that acceleration of CRs proceeds up to PeV energies has been rejected in all known young SNRs because of the clear cutoffs detected at several TeVs in their spectra.

The GeV-to-TeV radiation from young shell-type SNRs, which had been long expected to provide final evidence to settle the question of the origin of CRs, can be either of hadronic or leptonic origin. Typically, a $1 \mathrm{TeV} \gamma$-ray photon is emitted either by an electron or a proton of about $10 \mathrm{TeV}$. The cooling time for $10 \mathrm{TeV}$ electrons is $\approx 5 \times 10^{4}$ $\mathrm{yr}$ (for IC scattering off the CMB photons) whereas for protons the cooling time is $5 \times 10^{7}\left(\mathrm{n} / 1 \mathrm{~cm}^{-3}\right)^{-1} \mathrm{yr}$ (see e.g.Aharonian 2004). Thus, the ratio of the luminosity in IC $\gamma$-rays to $\pi^{0}$-decay $\gamma$-rays is of the order of $10^{3} \times\left(W_{e} / W_{p}\right) \times\left(n / 1 \mathrm{~cm}^{-3}\right)^{-1}$. The leptonic contribution to the emission is thus dominant over the hadronic one unless $W_{e} / W_{p} \ll 10^{-3}$ or, alternatively, if the gas density inside the shell is $n \gg 1 \mathrm{~cm}^{-3}$. This latter condition, however, if realised, would have the drawback of slowing down the shock wave very quickly and thus prevent efficient acceleration. On the other hand, a much larger energy density in protons than in electrons could result from rapid cooling of the electron population, especially in the presence of an amplified magnetic field, as inferred for young SNRs. If $B>10 \mu \mathrm{G}$, only a small fraction of their energy $w_{M B R} / w_{B} \approx$ $0.1(B / 10 \mu \mathrm{G})^{-2}$, is released in IC $\gamma$-rays, making the conditions for detection of $\pi^{0}$ decay more favourable.

To recap, no known SNR has proved to accelerate particles beyond $100 \mathrm{TeV}$. In addition, the power in accelerated protons within SNRs derived from $\gamma$-ray observations depends on the highly uncertain local gas density $n$ and on the magnetic and radiation fields within the remnant. As a result, it has so far been impossible to unequivocally prove that the population of SNRs in the Galaxy injects the necessary power (about $10^{50} \mathrm{erg}$ per SN event) to sustain the CR population. In fact simulations of the SNR population show how the choice of acceleration and ISM parameters lead to remarkably different SNR populations as CR sources (Cristofari et al. 2017).

Some other classes of astrophysical sources, such as superbubbles or star-forming regions (see § 5.2) or remnants of GRBs in our Galaxy (Atoyan, Buckley \& Krawczynski 2006), and, in particular, the centre of our Galaxy (see $§ 5.1$ ), have long been proposed as alternative accelerators of particles and major contributors to the population of Galactic CRs up to PeV energies (Casse \& Paul 1980; Voelk \& Forman 1982; Cesarsky \& Montmerle 1983; Bykov \& Toptygin 2001; Parizot et al. 2004; Torres, Domingo-Santamaría \& Romero 2004; Higdon \& Lingenfelter 2005; Atoyan et al. 2006; Reimer, Pohl \& Reimer 2006; Aharonian, Yang \& de Oña Wilhelmi 2019).

\subsection{Galactic Centre}

A breakthrough in the quest for the origin of the highest energy CRs in the Galaxy was the discovery of a powerful PeVatron in the centre of the Milky Way (H.E.S.S. Collaboration et al. 2016). 
The nucleus of the Milky Way is a very active region with numerous sources of non-thermal radiation and constitutes a unique laboratory for the study of VHE astrophysical processes within the Galaxy and in external active galactic nuclei. The Galactic Centre (GC) is thought to host a super-massive black hole (SMBH) of $2.6 \times 10^{6}$ Solar masses (Ghez et al. 2008; Gillessen et al. 2009) located very close to the dynamical centre of the Galaxy and coincident with the compact radio source Sagittarius A* (Sgr $\left.\mathrm{A}^{*}\right)$. Sgr A* emits radiation in X-rays and infrared through accretion of mass onto the $\mathrm{BH}$. The region within $400 \mathrm{pc}$ from the GC, called the Central Molecular Zone (CMZ), contains around $5 \%$ of the total Galactic molecular gas (Oka et al. 1998; Tsuboi, Handa \& Ukita 1999). The molecular CO line towards the GC being somehow optically thick due to the high gas density, the gas distribution there is mapped additionally with other lines, such the CS radio line (Tsuboi et al. 1999). Although the presence of this dense gas and dust prevents observations of this region in the optical and ultraviolet wavelengths, the GC region is extremely bright at radio, infrared, X-ray and $\gamma$-ray frequencies.

At VHE (Abdalla et al. 2018), the GC hosts a bright point-like source, HESS J1745-290, which, within errors, coincides spatially with Sgr A* and presents an energy spectrum with a steepening below $10 \mathrm{TeV}$. While the association of HESS J1745-290 with Sgr A* is well motivated in terms of spatial coincidence and of required energetics, the pulsar wind nebula (PWN) candidate G359.95-0.04 is also a viable counterpart of HESS J1745-290. HESS J1745-290 is surrounded by an extended component of VHE $\gamma$-ray emission, correlated spatially with the CMZ. The spectrum of this radiation is a pure power law, with a spectral index -2.3 and without evidence of a spectral cutoff.

Owing to their different energy distributions, the central point-like source and the extended emission cannot have the same origin. The diffuse VHE $\gamma$-ray emission of the $\mathrm{CMZ}$ could, in principle, be produced through interactions of either relativistic protons with the ambient gas or of relativistic electrons with the radiation fields. However, a leptonic origin of the diffuse $\gamma$-rays can be excluded. The power-law acceleration spectra of electrons should extend to about $100 \mathrm{TeV}$, which is extremely difficult because of the severe IC and synchrotron radiative losses in the GC region. For the same reason, leptons hardly can escape the sites of their acceleration and propagate over the tens of parsec extended region of the HESS diffuse emission.

The spatial correlation between the $\gamma$-ray emission from the Galactic Ridge and the ambient gas supports a hadronic origin of the emission. The spectrum of the parental protons, with a spectral index close to -2.4 , should extend to energies close to $1 \mathrm{PeV}$. Assuming a cutoff in the parent proton spectrum, the corresponding secondary $\gamma$-ray spectrum deviates from the HESS data at $68 \%, 90 \%$, and $95 \%$ confidence levels for cutoffs at 2.9, 0.6 and $0.4 \mathrm{PeV}$, respectively. This makes the discovery of the diffuse emission from the GC the first robust claim of detection of a Galactic CR PeVatron.

The CR energy density in the CMZ, obtained combining the $\gamma$-ray and gas distributions, is an order of magnitude higher than the sea of CRs. The radial distribution of CRs follows a $1 / r$ dependence, where $r$ is the distance to the GC. This means that the PeVatron should be located within $10 \mathrm{pc}$ from the GC and the total energy output in protons above $10 \mathrm{TeV}$ over the whole region should amount to $\mathrm{W}_{\mathrm{CR}}=10^{49} \mathrm{erg}$. Such a modest energy output could be provided by a single SNR event, such as Sgr A East, a Pulsar Wind Nebula. If the SMBH, Sgr A*, is the GC PeVatron, then the particles producing the HESS extended emission are accelerated either in the vicinity of the SMBH, close to event horizon, or at the termination of a relativistic outflow, either a jet or a wind. This jet or wind should be injected close to the black hole and carry a substantial fraction of energy, extracted from the accretion disk. Indeed, the power required by the inferred CR distribution corresponds to $\gtrsim 1 \%$ of the accretion power of the central black hole, and is $2-3$ orders of magnitude 
larger than the bolometric luminosity of Sgr A*. The source, however, could have been more active in the past (H.E.S.S. Collaboration et al. 2016).

Alternatively, the extended $\mathrm{TeV}$ emission from the GC could be the combined result of acceleration within three powerful star clusters, the Arches, the Quintuplet and the Nuclear cluster (H.E.S.S. Collaboration et al. 2016; Aharonian et al. 2019). In this case, the continuous injection over millions of years, required by the $1 / r$ distribution of the diffuse emission, could result from the characteristic ages of massive clusters, roughly $10^{6}$ years (Aharonian et al. 2019).

All the proposals discussed here assume that particle transport in the GC vicinity can be described through the same simple model of a spatially uniform diffusion coefficient adopted for the rest of the Galaxy. If this assumption is released, however, the GC excess can be interpreted as a result of a spatially dependent diffusion coefficient, as was proposed by Gaggero et al. (2017), based on Fermi-LAT data showing the spatial dependence of the $\gamma$-ray galactic diffuse emission.

\subsection{Star clusters}

Collective stellar winds and SNR shocks in clusters and associations of massive stars have long been suggested as possible, alternative or additional contributors to the Galactic CR flux (Casse \& Paul 1980; Voelk \& Forman 1982; Cesarsky \& Montmerle 1983). Core-collapse SN progenitor stars and colliding wind binaries evolve in giant molecular clouds and mostly remain close to their birthplaces in groups of loosely bound associations or dense stellar clusters. The winds of multiple massive stars in such systems can collide and form collective cluster winds which drive a giant bubble, a so-called superbubble, filled with a hot $\left(T=10^{6} \mathrm{~K}\right)$ and tenuous $\left(n<0.01 \mathrm{~cm}^{-3}\right)$ plasma. At the termination shock of the stellar cluster wind, turbulence can build up, in the form of MHD fluctuations and weak shocks (Bykov \& Toptygin 2001; Torres et al. 2004; Reimer et al. 2006). Turbulence in the superbubble interiors can accelerate particles to VHEs, not only through the first-order Fermi process, but also via the second-order mechanism (Bykov \& Toptygin 2001). SN explosions of massive stars in thin and hot superbubbles can also produce efficient particle acceleration at the boundary of the superbubbles or at MHD turbulence and further amplify existing MHD turbulence (Ferrand \& Marcowith 2010). It has also been recognised that multiple shocks can result in efficient acceleration beyond $\mathrm{PeV}$ energies (Klepach, Ptuskin \& Zirakashvili 2000). The interaction of the accelerated particles with the ambient medium, often including dense molecular clouds, or with electromagnetic fields, leads to the efficient production of VHE $\gamma$-rays.

Recent observations at $\mathrm{TeV}$ energies of massive star-forming regions and stellar clusters, such as 30 Doradus in the LMC (H.E.S.S. Collaboration et al. 2015), Westerlund 1 (Abramowski et al. 2012) and the Cygnus region (Aharonian et al. 2002; Abdo et al. 2007; Konopelko et al. 2007; Abdo et al. 2009; Bartoli et al. 2012) in our own Galaxy, support the hypothesis that star forming regions are sites of high-energy particle acceleration, and give new impulse to the $\gamma$-ray research in this field. The primary objectives of these $\gamma$-ray observations are: (1) to constrain the fraction of mechanical energy of the stellar wind transferred to relativistic particles and, hence, $\gamma$-rays; (2) to unveil the physics of particle acceleration and propagation in Galactic stellar clusters and superbubbles. Furthermore, high-energy phenomena are also receiving increasing attention from the point of view of their impact on the life cycle of interstellar matter and star-formation processes. The rate and efficiency of the star formation process depends, in fact, on the balance between the self-gravity of dense molecular cores and the countervailing forces which act to support the clouds. The most important of these are likely to be thermal pressure, turbulence and magnetic fields. In order for magnetic support to be effective, a population of ionised 
particles must be present in the core. As molecular clouds are opaque to ultraviolet radiation from stars, the main ionising agent is thought to be low-energy CRs, and the magnetic support of the cloud is critically dependent on their abundance.

The Cygnus region hosts some of the most remarkable star-forming systems in the Milky Way, including Cygnus X, a star-forming region at only $1.5 \mathrm{kpc}$ from the Sun, with a total mass in molecular gas of a few million Solar masses, at least 10 times the total mass in all other close-by star-forming regions, such as Carina or Orion, and a total mechanical stellar wind energy input of $10^{39} \mathrm{erg} \mathrm{s}^{-1}$, which corresponds to several per cent of the kinetic energy input by SNs in the entire Galaxy. Cygnus $\mathrm{X}$ hosts many young star clusters and several groups of $\mathrm{O}$ - and B-type stars, called OB associations. One of these associations, Cygnus OB2, contains 65 O stars and nearly 500 B stars. These super stars have created cavities filled with hot, thin gas surrounded by ridges of cool, dense gas where stars are now forming, which strongly emit at GeV energies, called the Fermi Cocoon (Ackermann et al. 2011). At $\mathrm{TeV}$ energies the Cygnus region shows two distinctive regions. One is possibly connected to the Cygnus $\mathrm{X}$ complex, the star association Cygnus OB2 and the Fermi Cocoon observed at $\mathrm{GeV}$ energies. In the other region detected at $\mathrm{TeV}$ energies, the Cygnus OB1 region, the Milagro collaboration discovered MGRO J2019+37 (Abdo et al. 2007), a very hard and extended source, possibly related to the massive star-forming region associated with the HII region Sharpless 104 (Sh 2-104) (Torres et al. 2004). Particle acceleration in shocks driven by the winds from the Wolf-Rayet stars in the young cluster Berk 87 in the Cygnus OB1 association have also been proposed as a possible origin of the VHE $\gamma$-rays (Bednarek 2007; Bednarek \& Sitarek 2007). VERITAS resolved the Milagro source into two sources, one of which, VER J2019+378, is a bright, $1^{\circ}$ extended source, that likely accounts for the bulk of the Milagro emission, coincident with the star formation region Sh 2-104. Its spectrum in the range $1-30 \mathrm{TeV}$ is well fitted with a power-law model of photon index 1.75, among the hardest values measured in the VHE band. The TeV counterpart of the Fermi Cocoon has been studied with the ARGO detector up to about 10 $\mathrm{TeV}$ and with the HAWC detector up to $200 \mathrm{TeV}$ (Bartoli et al. 2014; Hona, Fleischhack \& Huentemeyer 2019). The spectral energy distribution shows a significant softening at a few TeV: this is revealed by the comparison between the ARGO and HAWC data and the Fermi-LAT data. This break in the $\gamma$-ray spectrum might hint at a cutoff in the injected CR spectrum (Hona et al. 2019), or possibly be explained as due to suppressed diffusion in the high-turbulence environment of the Cygnus superbubble, which would confine low-energy particles whereas higher-energy particles escape. Aharonian et al. (2019) argued that three ultra-compact clusters located in the GC power the HESS diffuse emission from the CMZ. The $1 / r$ dependence of the CR density on distance from the star cluster is, in fact, a distinct signature of continuous injection of CRs over the cluster lifetime and following diffusion through ISM. The efficiency of conversion of kinetic energy of powerful stellar winds can be as high as $10 \%$. This implies that the population of young massive stars can provide production of CRs at a rate of up to $10^{41} \mathrm{erg} \mathrm{s}^{-1}$, which is sufficient to support the flux of Galactic CRs without invoking other source populations. This GC PeVatrons together with the other massive star-forming regions, such as Westerlund 1 and Cygnus OB2, would represent the major factories of Galactic CRs (Aharonian et al. 2019). A broader review of this subject, including a discussion of the subtleties associated with acceleration and propagation of CRs in these environments, may be found in Bykov et al. (2020).

\section{Implications of anti-matter data and CR leptons}

One final subject we want to address in this paper concerns the implications of recent observational results on the spectrum of CR electrons, positrons and anti-protons. Although electrons can be directly accelerated from the ISM plasma, positrons and 
anti-protons are extremely low in number in the ordinary ISM and, hence, they have long been considered to be purely secondaries, namely a byproduct of CR interactions during propagation in the Galaxy. If one assumes that $e^{+}$and $\bar{p}$ are pure secondaries, the straightforward expectation is that the ratio between their fluxes and that of protons (their primary parent particles) should monotonically decrease with energy, as is true for the $\mathrm{B} / \mathrm{C}$ ratio discussed in $\S 4$. In fact, the ratio between $e^{+}$and $p$ should decrease even faster, because positrons are subject to radiation losses which further steepen the spectrum at high energies (see e.g.Amato \& Blasi (2018) for extended discussion). Direct observations of the flux of positrons (Adriani et al. 2009; Aguilar et al. 2013) and anti-protons (Aguilar et al. 2016a) contradict this expectation, showing that the spectra of $e^{+}, \bar{p}$ and $p$ are all parallel.

Several different scenarios have been proposed in the literature to explain this finding (e.g.Blum, Katz \& Waxman 2013; Cowsik, Burch \& Madziwa-Nussinov 2014; Cowsik \& Madziwa-Nussinov 2016; Eichler 2017; Lipari 2017). An interesting possibility is that CRs accumulate sizeable grammage in the vicinity of their sources, where the diffusion coefficient is reduced with respect to the Galactic average. Here diffusion is energy dependent and this is where most of the boron is produced. Then, in the rest of the Galaxy, diffusion must be energy independent and faster than usually assumed. Most $e^{+}$and $\bar{p}$ would be produced by particles that accumulate most of their grammage in this second region: at a given rigidity their parent particles are $\sim 10$ times more energetic than those that produce B nuclei, and hence spend a shorter time in the source vicinity.

Although it is not fully clear that these assumptions would not violate other constraints, such as those provided by anisotropy, a possible physical justification of such a scenario could stem from the effects of self-generated turbulence (see $\S 3$ ) which could considerably reduce the diffusion coefficient in the vicinity of CR sources. In terms of theory the viability of this scenario is not fully clear, especially due to the unknowns related to the abundance of neutrals, which can effectively damp the CR-induced resonant streaming instability (D’Angelo, Blasi \& Amato 2016; Nava et al. 2016). However, there are observational indications that enhanced confinement indeed occurs, at least in the vicinity of some sources. A striking example are the so-called $\mathrm{TeV}$ halos (Abeysekara et al. 2017) surrounding evolved pulsars. Many more cases are expected to be found with next-generation $\gamma$-ray telescopes, such as CTA.

In reality, given the present uncertainties in many of the parameters involved, among which, most notably, the $\bar{p}$ production cross-section (Korsmeier, Donato \& Di Mauro $2018)$, the $\bar{p} / p$ ratio is not in a statistically significant disagreement with the standard description of $\mathrm{CR}$ propagation through the Galaxy, nor with the $\mathrm{B} / \mathrm{C}$ ratio, when all available information is taken into account and the subtle effects of re-acceleration are included in the description (Bresci et al. 2019). Indeed the high precision of the currently available data prompts theory to move forward and include second-order effects: one of these is the finite probability that during propagation particles might encounter an active CR accelerator (e.g. a SNR shock) and be re-accelerated. This has no effect on $\mathrm{CR}$ primaries, but flattens the spectrum of CR secondaries. Taking this phenomenon into account, together with the hardening of primaries, the energy dependence of the $\bar{p}$ production cross-section and, finally, the source grammage mentioned in $\S 4$, allows one to reproduce the $\mathrm{B} / \mathrm{C}$ ratio, the $\bar{p} / p$ ratio and all available data on stable nuclei (Bresci et al. 2019).

Differently from $\bar{p}$, the spectrum of $e^{+}$is not easy to reproduce in the standard CR propagation scenario, unless additional sources of primary positrons are invoked.

The leptonic CR population is a small fraction of the total CR population. For instance at $\sim 1 \mathrm{GeV}$ the ratio of the hadronic versus leptonic fluxes measured at Earth is roughly 
(a)

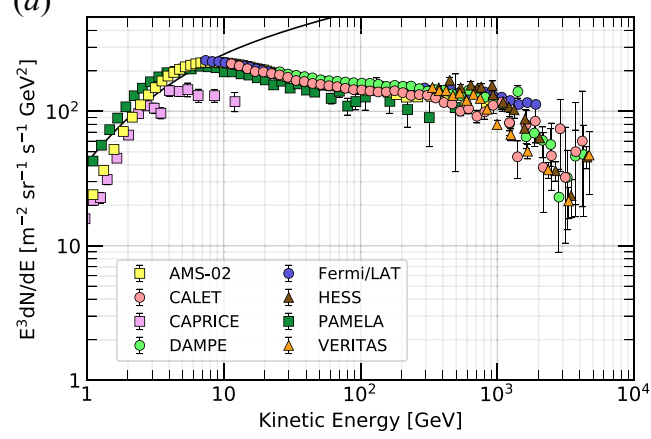

(b)

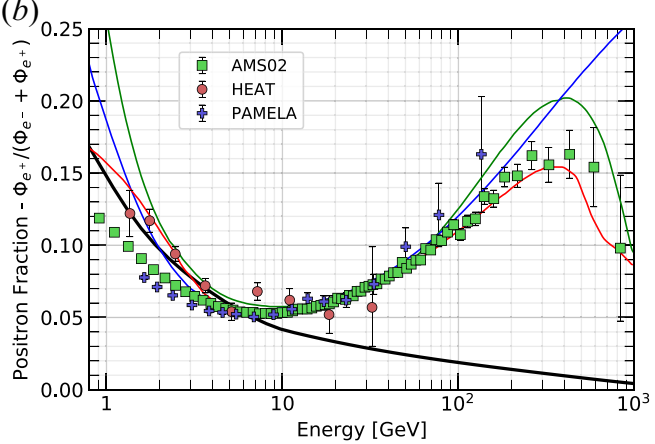

FIGURE 4. (a) Differential spectrum of electrons and positrons multiplied by $E^{3}$. CR electrons can be measured using spaceborne instruments such as AMS or Fermi-LAT up to $1 \sim \mathrm{TeV}$ and CALET or DAMPE up to $\sim 10 \mathrm{TeV}$. Ground-based Cherenkov telescopes, such as H.E.S.S., MAGIC and VERITAS, which benefit from very large effective areas, have measured the flux up to $20 \mathrm{TeV}$. The black line shows the proton spectrum multiplied by 0.01 (Shikaze et al. 2007).

(b) Positron fraction. Not all experiments are able to measure the electrons and positrons separately. Here measurements by Aguilar et al. (2019a), Adriani et al. (2009) and Beatty et al. (2004) are reported, together with a number of model predictions: the black curve is for pure secondary production in the standard scenario (Moskalenko \& Strong 1998); the blue curve includes a more sophisticated propagation model (Gaggero et al. 2013); the green curve invokes dark matter decay (Ibarra, Tran \& Weniger 2013); the red curve includes a contribution from pulsars (Yin et al. 2013). Figure reproduced from Particle Data Group et al. (2020).

$100: 1$ (https://lpsc.in2p3.fr/crdb/). Differently from hadrons, leptons are heavily affected by energy losses owing to synchrotron and IC processes in the interstellar magnetic and radiation fields. If positrons were purely secondary products of $\mathrm{CR}$ interactions, their spectrum would have to be steeper than that of protons at energies where losses are important. As recently shown by Diesing \& Caprioli (2020), in order for this not to happen, the escape time from the Galaxy would have to be so short that protons would not be able to produce the observed $\bar{p}$ flux.

The anomalous positron spectrum has been at the origin of a huge number of papers, proposing either astrophysical or particle physics (dark matter annihilation-related) solutions (Aguilar et al. 2019b). Limiting the discussion to astrophysical scenarios, important constraints on the plausibility of the different proposals come from the maximum age and distance of the highest-energy lepton sources. As shown in figure 4, electrons and positrons are now detected up $20 \mathrm{TeV}$. The electron spectrum shows roughly a constant slope up to an energy of about $1 \mathrm{TeV}$, above which the spectrum becomes steeper. One possibility to interpret the break at $1 \mathrm{TeV}$ is to relate it to the transition between a regime where a large number of lepton accelerators contribute to the spectrum, to a regime where only a few or even only one single electron source very close to the Sun contributes (Recchia et al. 2019).

The number of contributing sources depends, in turn, on the average intensity of the magnetic and radiation fields and on the diffusion coefficient in the Galaxy. The latter, in particular, seems to need some revision in light of the recent measurements of secondary nuclei, both stable and unstable, provided by AMS-02 (Aguilar et al. 2018).

For the standard average values of the magnetic and radiation fields in the Galaxy, the cooling time of leptons with energies above few tens of $\mathrm{GeV}$ can be approximately 
estimated as

$$
t_{\mathrm{cool}} \approx 10^{6}\left[\left(\frac{U_{\mathrm{rad}}}{0.3 \mathrm{eV} \mathrm{cm}^{-3}}\right)+\left(\frac{B}{3 \mu \mathrm{G}}\right)^{2}\right]^{-1}\left(\frac{E}{1 \mathrm{TeV}}\right)^{-1}
$$

Losses limit the distance $\lambda$ from which leptons of a given energy can reach us to $\lambda \approx$ $2 \sqrt{D(E) t_{\text {cool }}}(E)$, with $D(E)$ the galactic diffusion coefficient.

Using for $D(E)$ the estimate provided by Evoli et al. (2020b), which allows all the available AMS-02 data on both primary and secondary nuclei (both stable and unstable) to be reproduced, one can estimate that the highest-energy leptons detected at the Earth cannot come from further than $\lambda(1 \mathrm{TeV}) \approx 3.5 \mathrm{kpc}$. This is a much larger distance than typically estimated in the past (see e.g. Atoyan, Aharonian \& Völk 1995) and ensures that many sources contribute to the lepton flux even at the highest energies (Evoli et al. 2020a).

The most promising sources of primary positrons are likely PWNs. These are the nebulae formed by the highly relativistic magnetised wind produced by a fast spinning, highly magnetised neutron star (see e.g. Amato (2019) for a recent review). The magnetosphere of such a star is a very efficient anti-matter factory and the pulsar wind contains electrons and positrons in about equal amounts. At the wind termination shock, these particles are accelerated up to PeV energies with a spectrum in the form of a broken power law, harder than $E^{-2}$ at energies below $\approx 500 \mathrm{GeV}$ and softer than $E^{-2}$ at higher energies. As discussed by Bykov et al. (2017), the accelerated particles are released in the ISM after the pulsar leaves its associated SNR to become a bow shock PWN. Once taken into account, the contribution of CR leptons from these sources allows to very well explain both the overall flux of leptons and the positron fraction with very reasonable values of the few free parameters (Evoli et al. 2020a).

\section{Conclusions and outlook}

In this article, we have tried to review some recent theoretical and observational developments in the quest for the origin of galactic CRs. High-energy observations, in the $\mathrm{X}$-rays, $\gamma$-rays and VHE $\gamma$-rays, have opened new possibilities to test the long-standing paradigm that wants these particles mainly produced in the blast waves of SN explosions.

X-ray observations have provided undisputable proof that SNRs can accelerate electrons up to tens of $\mathrm{TeV}$, and at the same time have shown the presence of amplified magnetic fields in their environment. These magnetic fields might be taken as a hint of efficient particle acceleration, although alternatives are possible. Their strengths are certainly in the range needed to make SNRs act as PeVatrons, accelerating particles up to the knee energy, where the galactic CR proton spectrum is expected to end. Despite this, theory points to a rare occurrence and an extremely short duration of the PeVatron phase for SNRs $(\S 3)$.

Multi-TeV photons (10-100 TeV photons) are the most direct messengers of the presence of protons in the critical knee region. Secondary electrons, produced as decay products of charged pions, may provide complimentary information through their X-ray synchrotron emission, but this channel has a lower radiative efficiency and secondary leptons are often subdominant with respect to primaries. In the end, the spectrum of the highest-energy $\gamma$-rays qualifies as the best source of information about the extension of the parent proton spectrum, and hence is the only direct tool to identify the PeVatrons and obtain essential information about the physics of particle acceleration and the formation of the knee.

Detailed $\gamma$-ray studies of young objects in this class have failed to show clear evidence of acceleration above $\sim 100 \mathrm{TeV}(\$ 2.1)$. Clear evidence of accelerated hadrons has only been found in middle-aged SNRs interacting with molecular clouds. The wealth of target 
for nuclear interactions makes these objects ideal candidates to be bright in $\gamma$-rays of hadronic origin, without contamination from leptonic IC emission. However, these sources are not very efficient as accelerators and the maximum energy they achieve is limited in all cases to $\lesssim 10 \mathrm{TeV}(\$ 2.2)$.

At the same time, observations in the $\mathrm{TeV}$ band have highlighted the existence of other kinds of sources showing relatively hard $\gamma$-ray spectra extending above 100 $\mathrm{TeV}$, in association with the GC region ( $\$ 5.1)$ and with young stellar clusters $(\S 5.2)$. How propagation in these complex environments, very likely more turbulent than the average ISM in the Galaxy, affects the spectrum finally released is yet to be thoroughly investigated.

On the other hand, direct measurements of CRs, and in particular high statistics spectra of primary and secondary nuclei, have provided new important insights on the properties of $\mathrm{CR}$ transport in the Galaxy, highlighting the presence of a change in propagation properties in the energy range $200-300 \mathrm{GeV}$. A possible interpretation of this finding is that below such energy CRs are mostly scattered by self-generated turbulence, another bit of information that stresses the importance of non-linearities in CR physics, and the importance of understanding how these particles affect not only the environment of their acceleration sites, but also the properties of the ISM at large ( $\$ 4)$.

Finally, a fundamentally important piece of information that we discussed has to do with the anti-matter component of CRs ( $\$ 6)$. Direct detection experiments showed that the leptonic anti-matter component of CRs can hardly be interpreted as of pure secondary origin. Additional sources of primary positrons seem to be required. The most natural candidates are pulsars and their nebulae, well known to be excellent positron factories. These will release their positrons only at late stages of their evolution, after leaving the parent SNRs. Crucial information about the particle release by these sources is likely to come from deeper studies of the $\gamma$-ray halos that have been found to surround some of them (Abeysekara et al. 2017).

In a time when a combination of new and surprising observations and theoretical difficulties are pushing the CR physics community to a deep re-evaluation of the SNR-CR connection, crucial tests are expected to come from high-energy astrophysical observations. All the recent surprises and issues that we have discussed through this article suggest that different astrophysical sources might contribute at different energies and at different levels to the CR population. This prompts to the need of a census of all possible CR contributors, a purpose that is well served by an unbiased survey of the Galactic Plane in the crucial multi-TeV energy range. Such survey is currently being undertaken with the high-altitude water Cherenkov detector, HAWC (Malone and HAWC Collaboration 2017). A PeVatron search conducted with detailed spectroscopy, higher sensitivity above $10 \mathrm{TeV}$ and higher angular resolution is a key scientific goal of the upcoming CTA (Cherenkov Telescope Array Consortium et al. 2019). In the search for PeVatrons in the Galaxy, CTA will benefit from a wider energy range and a much better angular resolution: although the latter will be essential to establish correlations with the gas maps and sources observed in other wavelengths, the former will finally give us access to the hundreds of $\mathrm{TeV}$ energy range, which is the realm where the hadronic mechanism is believed to be strongly dominant over the leptonic one.

\section{Acknowledgements}

We thank the anonymous reviewers for their careful reading of the manuscript and their many useful comments, which in our opinion have substantially improved the paper.

E.A. acknowledges support by INAF and ASI through Grants SKA-CTA INAF 2016, INAF-MAINSTREAM 2018 and ASI/INAF No. 2017-14-H.O and by the National Science 
Foundation under Grant No. NSF PHY-1748958. S.C. acknowledges the support from Polish Science Centre grant, DEC-2017/27/B/ST9/02272.

Editor William Dorland thanks the referees for their advice in evaluating this article.

\section{REFERENCES}

Aguilar, M., Aisa, D., Alpat, B., Alvino, A., Ambrosi, G., Andeen, K., Arruda, L., Attig, N., AzZarello, P., BAChlechner, A., et al. 2015a Precision measurement of the proton flux in primary cosmic rays from rigidity $1 \mathrm{GV}$ to $1.8 \mathrm{TV}$ with the alpha magnetic spectrometer on the International Space Station. Phys. Rev. Lett. 114, 171103.

Aguilar, M., Aisa, D., Alpat, B., Alvino, A., Ambrosi, G., Andeen, K., Arruda, L., Attig, N., Azzarello, P., Bachlechner, A., et al. 2015b Precision measurement of the helium flux in primary cosmic rays of rigidities $1.9 \mathrm{GV}$ to $3 \mathrm{TV}$ with the alpha magnetic spectrometer on the International Space Station. Phys. Rev. Lett. 115 (21), 211101.

Aguilar, M., Alberti, G., Alpat, B., Alvino, A., Ambrosi, G., Andeen, K., Anderhub, H., Arruda, L., AzZArello, P., BACHlechner, A., et al. 2013 First result from the alpha magnetic spectrometer on the International Space Station: precision measurement of the positron fraction in primary cosmic rays of 0.5-350 GeV. Phys. Rev. Lett. 110 (14), 141102.

Abdo, A.A., Ackermann, M., Ajello, M., Allafort, A., Baldini, L., Ballet, J., Barbiellini, G., Baring, M.G., BAstieri, D., Bellazzini, R., et al. 2011 Observations of the young supernova remnant RX J1713.7-3946 with the Fermi large area telescope. Astrophys. $J$. 734 (1), 28. arXiv:1103.5727.

Abdo, A.A., Ackermann, M., Ajello, M., Baldini, L., Ballet, J., Barbiellini, G., Baring, M.G., Bastieri, D., Baughman, B.M., Bechtol, K., et al. $2010 a$ Gamma-ray emission from the shell of supernova remnant W44 revealed by the Fermi LAT. Science 327 (5969), 1103.

Abdo, A.A., Ackermann, M., Ajello, M., Baldini, L., Ballet, J., Barbiellini, G., Bastieri, D., Baughman, B.M., Bechtol, K., Bellazzini, R., et al. $2010 b$ Observation of supernova remnant IC 443 with the Fermi Large Area Telescope. Astrophys. J. 712 (1), 459-468. arXiv: 1002.2198 .

Abdo, A.A., Allen, B.T., Aune, T., Berley, D., Chen, C., Christopher, G.E., DeYoung, T., Dingus, B.L., Ellsworth, R.W., GonZalez, M.M., et al. 2009 Milagro observations of multi-TeV emission from galactic sources in the Fermi bright source list. Astrophys. J. Lett. 700 (2), L127-L131. arXiv:0904.1018.

Abdo, A.A., Allen, B., Berley, D., Casanova, S., Chen, C., Coyne, D.G., Dingus, B.L., Ellsworth, R.W., Fleysher, L., Fleysher, R., et al. 2007 TeV gamma-ray sources from a survey of the galactic plane with Milagro. Astrophys. J. Lett. 664 (2), L91-L94. arXiv:0705.0707.

Abeysekara, A.U., Albert, A., Alfaro, R., Alvarez, C., Álvarez, J.D., Arceo, R., Arteaga-Velázquez, J.C., Avila Rojas, D., Ayala Solares, H.A., Barber, A.S., et al. 2017 Extended gamma-ray sources around pulsars constrain the origin of the positron flux at Earth. Science 358 (6365), 911-914. arXiv:1711.06223.

Abramowski, A., Acero, F., Aharonian, F., Akhperjanian, A.G., Anton, G., Balzer, A., Barnacka, A., BArRes de Almeida, U., Becherini, Y., BeCKer, J., et al. 2012 Discovery of extended VHE $\gamma$-ray emission from the vicinity of the young massive stellar cluster Westerlund 1. Astron. Astrophys. 537, A114. arXiv:1111.2043.

Acciari, V.A., Aliu, E., Arlen, T., Aune, T., Bautista, M., Beilicke, M., Benbow, W., Bradbury, S.M., BuCKLEY, J.H., Bugaev, V., et al. 2009 Observation of extended very high energy emission from the supernova remnant IC 443 with VERITAS. Astrophys. J. Lett. 698 (2), L133-L137. arXiv:0905.3291.

Acciari, V.A., Aliu, E., Arlen, T., Aune, T., Beilicke, M., Benbow, W., Bradbury, S.M., Buckley, J.H., Bugaev, V., Byrum, K., et al. 2011 Discovery of TeV gamma-ray emission from Tycho's supernova remnant. Astrophys. J. Lett. 730 (2), L20. arXiv:1102.3871. 
Ackermann, M., Ajello, M., Allafort, A., Baldini, L., Ballet, J., Barbiellini, G., Bastieri, D., Belfiore, A., Bellazzini, R., Berenji, B., et al. 2011 A cocoon of freshly accelerated cosmic rays detected by fermi in the cygnus superbubble. Science 334 (6059), 1103.

Adriani, O., Barbarino, G.C., Bazilevskaya, G.A., Bellotti, R., Boezio, M., Bogomolov, E.A., Bonechi, L., Bongi, M., Bonvicini, V., Bottai, S., et al. 2009 An anomalous positron abundance in cosmic rays with energies 1.5-100 GeV. Nature 458 (7238), 607-609. arXiv:0810.4995.

Adriani, O., Barbarino, G.C., Bazilevskaya, G.A., Bellotti, R., Boezio, M., Bogomolov, E.A., Bonechi, L., Bongi, M., Bonvicini, V., Borisov, S., et al. 2011 PAMELA measurements of cosmic-ray proton and helium spectra. Science 332 (6025), 69. arXiv:1103.4055.

Aguilar, M., Ali Cavasonza, L., Alpat, B., Ambrosi, G., Arruda, L., Attig, N., Aupetit, S., Azzarello, P., Bachlechner, A., BArao, F., et al. 2016 a Antiproton flux, antiproton-to-proton flux ratio, and properties of elementary particle fluxes in primary cosmic rays measured with the alpha magnetic spectrometer on the international space station. Phys. Rev. Lett. 117 (9), 091103.

Aguilar, M., Ali Cavasonza, L., Ambrosi, G., Arruda, L., Attig, N., Aupetit, S., Azzarello, P., Bachlechner, A., Barao, F., BArrau, A., et al. $2016 b$ Precision measurement of the boron to carbon flux ratio in cosmic rays from $1.9 \mathrm{GV}$ to $2.6 \mathrm{TV}$ with the alpha magnetic spectrometer on the international space station. Phys. Rev. Lett. 117 (23), 231102.

Aguilar, M., Ali Cavasonza, L., Ambrosi, G., Arruda, L., Attig, N., Aupetit, S., Azzarello, P., Bachlechner, A., Barao, F., Barrau, A., et al. 2018 Observation of new properties of secondary cosmic rays Lithium, Beryllium, and Boron by the alpha magnetic spectrometer on the international space station. Phys. Rev. Lett. 120 (2), 021101.

Aguilar, M., Ali Cavasonza, L., Alpat, B., Ambrosi, G., Arruda, L., Attig, N., Azzarello, P., Bachlechner, A., Barao, F., BArrau, A., et al. 2019a Towards understanding the origin of cosmic-ray electrons. Phys. Rev. Lett. 122, 101101.

Aguilar, M., Ali Cavasonza, L., Ambrosi, G., Arruda, L., Attig, N., Azzarello, P., BACHlechner, A., BARAO, F., BARraU, A., BARrin, L., et al. $2019 b$ Towards understanding the origin of cosmic-ray positrons. Phys. Rev. Lett. 122, 041102.

Aharonian, F.A. 2001 Gamma rays from molecular clouds. Space Sci. Rev. 99, 187-196. arXiv:astro-ph/0012290.

Aharonian, F.A. 2004 Very High Energy Cosmic Gamma Radiation: A Crucial Window on the Extreme Universe. World Scientific Publishing.

Aharonian, F.A., Akhperjanian, A.G., Aye, K.M., Bazer-Bachi, A.R., Beilicke, M., Benbow, W., Berge, D., Berghaus, P., Bernlöhr, K., Bolz, O., et al. 2004 High-energy particle acceleration in the shell of a supernova remnant. Nature 432 (7013), 75-77. arXiv:astro-ph/0411533.

Aharonian, F., Akhperjanian, A., Beilicke, M., Bernlöhr, K., Börst, H., Bojahr, H., Bolz, O., Coarasa, T., Contreras, J., Cortina, J., et al. 2002 An unidentified TeV source in the vicinity of Cygnus OB2. Astron. Astrophys. 393, L37-L40. arXiv:astro-ph/0207528.

AHARONIAN, F.A. \& ATOYAN, A.M. 1996 On the emissivity of $\pi$ 0-decay gamma radiation in the vicinity of accelerators of galactic cosmic rays. Astron. Astrophys. 309, 917-928.

Aharonian, F.A., Drury, L.O'C. \& VoelK, H.J. $1994 \mathrm{GeV} / \mathrm{TeV}$ gamma-ray emission from dense molecular clouds overtaken by supernova shells. Astron. Astrophys. 285, 645-647.

Aharonian, F., Peron, G., Yang, R., Casanova, S. \& Zanin, R. 2020 Probing the sea of galactic cosmic rays with fermi-lat. Phys. Rev. D 101, 083018.

Aharonian, F., YANG, R. \& DE OÑA Wilhelmi, E. 2019 Massive stars as major factories of Galactic cosmic rays. Nature Astron. 3, 561-567. arXiv:1804.02331.

Aharonian, F., Akhperjanian, A., Barrio, J., Bernlöhr, K., Börst, H., Bojahr, H., Bolz, O., Contreras, J., Cortina, J., Denninghoff, S., et al. 2001 Evidence for TeV gamma ray emission from Cassiopeia A. Astron. Astrophys. 370, 112-120. arXiv:astro-ph/0102391.

Ahn, H.S., Allison, P., Bagliesi, M.G., Beatty, J.J., Bigongiari, G., Childers, J.T., Conklin, N.B., Coutu, S., DuVernois, M.A., Ganel, O., et al. 2010 Discrepant hardening observed in cosmic-ray elemental spectra. Astrophys. J. Lett. 714 (1), L89-L93. arXiv:1004.1123. 
Ahnen, M.L., Ansoldi, S., Antonelli, L.A., Arcaro, C., Babić, A., Banerjee, B., Bangale, P., Barres de Almeida, U., BArrio, J.A., Becerra GonzÁlez, J., et al. 2017 A cut-off in the TeV gamma-ray spectrum of the SNR Cassiopeia A. Mon. Not. R. Astron. Soc. 472 (3), 2956-2962. arXiv:1707.01583.

Albert, J., Aliu, E., Anderhub, H., Antoranz, P., Armada, A., Baixeras, C., Barrio, J.A., BARtKo, H., BAstieri, D., Becker, J.K., et al. 2007a Observation of VHE $\gamma$-rays from Cassiopeia A with the MAGIC telescope. Astron. Astrophys. 474 (3), 937-940. arXiv:0706.4065.

Albert, J., Aliu, E., Anderhub, H., Antoranz, P., Armada, A., Baixeras, C., Barrio, J.A., BARtKo, H., BAstieri, D., BeCKer, J.K., et al. $2007 b$ Discovery of very high energy gamma radiation from IC 443 with the MAGIC telescope. Astrophys. J. Lett. 664 (2), L87-L90. arXiv:0705.3119.

Aloisio, R., Blasi, P. \& SERPICO, P.D. 2015 Nonlinear cosmic ray Galactic transport in the light of AMS-02 and Voyager data. Astron. Astrophys. 583, A95. arXiv:1507.00594.

AмAто, E. 2014 The origin of galactic cosmic rays. Intl J. Mod. Phys. D 23 (7), 1430013. arXiv:1406.7714.

AмAто, E. 2019 The theory of pulsar wind nebulae: recent progress. In High Energy Phenomena in Relativistic Outflows VII, p. 33. PoS - Proceedings of Science.

AмAтo, E. \& Blasi, P. 2006 Non-linear particle acceleration at non-relativistic shock waves in the presence of self-generated turbulence. Mon. Not. R. Astron. Soc. 371 (3), 1251-1258. arXiv:astro-ph/0606592.

AmAto, E. \& BLASI, P. 2009 A kinetic approach to cosmic-ray-induced streaming instability at supernova shocks. Mon. Not. R. Astron. Soc. 392 (4), 1591-1600. arXiv:0806.1223.

Amato, E. \& Blasi, P. 2018 Cosmic ray transport in the galaxy: a review. Adv. Space Res. 62 (10), 2731-2749. arXiv:1704.05696.

Ambrogi, L., Celli, S. \& Aharonian, F. 2018 On the potential of Cherenkov telescope arrays and KM3 neutrino telescopes for the detection of extended sources. Astropart. Phys. 100, 69-79. arXiv:1803.03565.

Archambault, S., Archer, A., Benbow, W., Bird, R., Bourbeau, E., Buchovecky, M., Buckley, J.H., Bugaev, V., Cerruti, M., Connolly, M.P., et al. 2017 Gamma-ray observations of Tycho's supernova remnant with VERITAS and fermi. Astrophys. J. 836 (1), 23. arXiv:1701.06740.

Atoyan, A.M., Aharonian, F.A. \& VöLK, H.J. 1995 Electrons and positrons in the galactic cosmic rays. Phys. Rev. D 52, 3265-3275.

Atoyan, A., BuCKLEy, J. \& KRAWCZYNSKI, H. 2006 A gamma-ray burst remnant in our galaxy: HESS J1303-631. Astrophys. J. Lett. 642 (2), L153-L156. arXiv:astro-ph/0509615.

BAADE, W. \& ZWICKY, F. 1934 Remarks on super-novae and cosmic rays. Phys. Rev. 46, 76-77.

Baghmanyan, V., Peron, G., Casanova, S., Aharonian, F. \& Zanin, R. 2020 Evidence of cosmic-ray excess from local giant molecular clouds. Astrophys. J. 901 (1), L4.

Bartoli, B., Bernardini, P., Bi, X.J., Bleve, C., Bolognino, I., Branchini, P., Budano, A., Calabrese Melcarne, A.K., Camarri, P., CAO, Z., et al. 2012 Observation of TeV gamma rays from the Cygnus region with the ARGO-YBJ experiment. Astrophys. J. Lett. 745 (2), L22. arXiv:1201.1973.

Bartoli, B., Bernardini, P., Bi, X.J., Branchini, P., Budano, A., Camarri, P., Cao, Z., Cardarelli, R., Catalanotti, S., Chen, S.Z., et al. 2014 Identification of the TeV gamma-ray source ARGO J2031+4157 with the Cygnus Cocoon. Astrophys. J. 790 (2), 152. arXiv:1406.6436.

Beatty, J.J., Bhattacharyya, A., Bower, C., Coutu, S., Duvernois, M.A., McKee, S., Minnick, S.A., Müller, D., Musser, J., Nutter, S., et al. 2004 New measurement of the cosmic-ray positron fraction from 5 to $15 \mathrm{GeV}$. Phys. Rev. Lett. 93 (24), 241102. arXiv:astro-ph/0412230.

BEDNAREK, W. $2007 \gamma$-ray production in young open clusters: Berk 87, Cyg OB2 and Westerlund 2. Mon. Not. R. Astron. Soc. 382, 367-376. arXiv:0704.3517.

BednareK, W. \& Sitarek, J. 2007 High-energy $\gamma$-rays from globular clusters. Mon. Not. R. Astron. Soc. 377, 920-930. arXiv:astro-ph/0701522.

Bell, A.R. 1978 The acceleration of cosmic rays in shock fronts - I. Mon. Not. R. Astron. Soc. 182, 147-156. 
BELL, A.R. 2004 Turbulent amplification of magnetic field and diffusive shock acceleration of cosmic rays. Mon. Not. R. Astron. Soc. 353 (2), 550-558.

Beresnyak, A., Jones, T.W. \& LAZARIAN, A. 2009 Turbulence-induced magnetic fields and structure of cosmic ray modified shocks. Astrophys. J. 707 (2), 1541-1549. arXiv:0908.2806.

BLANDFORD, R.D. \& OSTRIKER, J.P. 1978 Particle acceleration by astrophysical shocks. Astrophys. J. Lett. 221, L29-L32.

BLASI, P. 2013 The origin of galactic cosmic rays. Astron. Astrophys. Rev. 21, 70. arXiv:1311.7346.

Blasi, P. \& AMATO, E. $2012 a$ Diffusive propagation of cosmic rays from supernova remnants in the Galaxy. I: spectrum and chemical composition. J. Cosmol. Astropart. Phys. 2012 (1), 010. arXiv:1105.4521.

Blasi, P. \& Amato, E. $2012 b$ Diffusive propagation of cosmic rays from supernova remnants in the Galaxy. II: anisotropy. J. Cosmol. Astropart. Phys. 2012 (1), 011. arXiv:1105.4529.

Blasi, P., Amato, E. \& Serpico, P.D. 2012 Spectral breaks as a signature of cosmic ray induced turbulence in the Galaxy. Phys. Rev. Lett. 109 (6), 061101. arXiv:1207.3706.

BLUM, K., KATZ, B. \& WAXMAN, E. 2013 AMS-02 results support the secondary origin of cosmic ray positrons. Phys. Rev. Lett. 111 (21), 211101. arXiv:1305.1324.

Blumenthal, G.R. \& Gould, R.J. 1970 Bremsstrahlung, synchrotron radiation, and compton scattering of high-energy electrons traversing dilute gases. Rev. Mod. Phys. 42, 237-270.

Bresci, V., Amato, E., Blasi, P. \& Morlino, G. 2019 Effects of re-acceleration and source grammage on secondary cosmic rays spectra. Mon. Not. R. Astron. Soc. 488 (2), 2068-2078. arXiv:1904.10282.

Buck, T., Pfrommer, C., Pakmor, R., Grand, R.J.J. \& Springel, V. 2020 The effects of cosmic rays on the formation of Milky Way-mass galaxies in a cosmological context. Mon. Not. R. Astron. Soc. 497 (2), 1712-1737. arXiv:1911.00019.

Bykov, A.M., Amato, E., Petrov, A.E., Krassilchtchikov, A.M. \& Levenfish, K.P. 2017 Pulsar wind nebulae with bow shocks: non-thermal radiation and cosmic ray leptons. Space Sci. Rev. 207 (1-4), 235-290. arXiv:1705.00950.

Bykov, A.M., Ellison, D.C. \& Renaud, M. 2012 Magnetic fields in cosmic particle acceleration sources. Space Sci. Rev. 166 (1-4), 71-95. arXiv:1105.0130.

Bykov, A.M., Marcowith, A., Amato, E., Kalyashova, M.E., Kruijssen, J.M.D. \& WAXMAn, E. 2020 High-energy particles and radiation in star-forming regions. Space Sci. Rev. 216 (3), 42. arXiv:2003.11534.

BYKov, A.M., OsiPOV, S.M. \& ElLISON, D.C. 2011 Cosmic ray current driven turbulence in shocks with efficient particle acceleration: the oblique, long-wavelength mode instability. Mon. Not. R. Astron. Soc. 410 (1), 39-52. arXiv:1010.0408.

BYKov, A.M. \& TOPTYGin, I.N. 2001 A model of particle acceleration to high energies by multiple supernova explosions in OB associations. Astron. Lett. 27 (10), 625-633.

CAPRIOLI, D. \& SPITKOVSKY, A. 2014a Simulations of ion acceleration at non-relativistic shocks. I. Acceleration efficiency. Astrophys. J. 783 (2), 91. arXiv:1310.2943.

CAPRIOLI, D. \& SPITKOVSKY, A. 2014b Simulations of ion acceleration at non-relativistic shocks. II. Magnetic field amplification. Astrophys. J. 794 (1), 46. arXiv:1401.7679.

CAPRIOLI, D. \& SPITKOVSKY, A. 2014c Simulations of ion acceleration at non-relativistic shocks. III. Particle diffusion. Astrophys. J. 794 (1), 47. arXiv:1407.2261.

CArdillo, M., Amato, E. \& Blasi, P. 2015 On the cosmic ray spectrum from type II supernovae expanding in their red giant presupernova wind. Astropart. Phys. 69, 1-10. arXiv:1503.03001.

CArdillo, M., Amato, E. \& Blasi, P. 2016 Supernova remnant W44: a case of cosmic-ray reacceleration. Astron. Astrophys. 595, A58. arXiv:1604.02321.

Casanova, S., Aharonian, F.A., Fukui, Y., Gabici, S., Jones, D.I., Kawamura, A., Onishi, T., Rowell, G., SANO, H., ToriI, K., et al. 2010a Molecular clouds as cosmic-ray barometers. Publ. Astron. Soc. Japan 62, 769-777. arXiv:0904.2887.

Casanova, S., Jones, D.I., Aharonian, F.A., Fukui, Y., Gabici, S., Kawamura, A., Onishi, T., Rowell, G., SAno, H., ToriI, K., et al. $2010 b$ Modeling the gamma-ray emission produced by runaway cosmic rays in the environment of RX J1713.7-3946. Publ. Astron. Soc. Japan 62, 1127-1134. arXiv:1003.0379. 
Cassam-Chenaï, G., Decourchelle, A., Ballet, J., Sauvageot, J.-L., Dubner, G. \& GIACANI, E. 2004 XMM-Newton observations of the supernova remnant RX J1713.7-3946 and its central source. Astron. Astrophys. 427, 199-216.

CAsse, M. \& PAul, J.A. 1980 Local gamma rays and cosmic-ray acceleration by supersonic stellar winds. Astrophys. J. 237, 236-243.

Cesarsky, C.J. 1980 Cosmic-ray confinement in the galaxy. Astron. Astrophys. Rev. 18, $289-319$.

Cesarsky, C.J. \& Montmerle, T. 1983 Gamma-rays from active regions in the galaxy - the possible contribution of stellar winds. Space Sci. Rev. 36 (2), 173-193.

Cherenkov Telescope Array Consortium, Acharya, B.S., Agudo, I., Al Samarai, I., Alfaro, R., Alfaro, J., Alispach, C., Alves Batista, R., Amans, J.-P., Amato, E., et al. 2019 Science With the Cherenkov Telescope Array. World Scientific Publishing.

Cowsik, R., Burch, B. \& MAdziWA-Nussinov, T. 2014 The origin of the spectral intensities of cosmic-ray positrons. Astrophys. J. 786 (2), 124. arXiv:1305.1242.

Cowsik, R. \& MADZIWA-Nussinov, T. 2016 Spectral intensities of antiprotons and the nested leaky-box model for cosmic rays in the Galaxy. Astrophys. J. 827 (2), 119. arXiv:1505.00305.

Cristofari, P., Blasi, P. \& Amato, E. 2020 The low rate of Galactic pevatrons. Astropart. Phys. 123, 102492. arXiv:2007.04294.

Cristofari, P., Gabici, S., Humensky, T.B., Santand er, M., Terrier, R., Parizot, E. \& CASANOVA, S. 2017 Supernova remnants in the very-high-energy gamma-ray domain: the role of the Cherenkov telescope array. Mon. Not. R. Astron. Soc. 471 (1), 201-209. arXiv:1709.01102.

Crutcher, R.M. 2012 Magnetic fields in molecular clouds. Annu. Rev. Astron. Astrophys. 50, $29-63$.

Cummings, A.C., Stone, E.C., Heikkila, B.C., Lal, N., Webber, W.R., Jóhannesson, G., Moskalenko, I.V., Orlando, E. \& Porter, T.A. 2016 Galactic cosmic rays in the local interstellar medium: Voyager 1 observations and model results. Astrophys. J. 831 (1), 18.

Dame, T.M., Hartmann, D. \& Thaddeus, P. 2001 The Milky Way in molecular clouds: a new complete CO survey. Astrophys. J. 547 (2), 792-813. arXiv:astro-ph/0009217.

D’Angelo, M., Blasi, P. \& AmAto, E. 2016 Grammage of cosmic rays around Galactic supernova remnants. Phys. Rev. D 94 (8), 083003. arXiv:1512.05000.

Diesing, R. \& CAPRIOLI, D. 2020 Nonsecondary origin of cosmic ray positrons. Phys. Rev. D 101 (10), 103030. arXiv:2001.02240.

Donnert, J., VAZZA, F., BrüGgen, M. \& ZuHone, J. 2018 Magnetic field amplification in galaxy clusters and its simulation. Space Sci. Rev. 214 (8), 122. arXiv:1810.09783.

Drury, L.O'C., Aharonian, F.A. \& VoelK, H.J. 1994 The gamma-ray visibility of supernova remnants. A test of cosmic ray origin. Astron. Astrophys. 287, 959-971. arXiv:astro-ph/9305037.

EICHLER, D. 2017 An alternative explanation of the varying boron-to-carbon ratio in galactic cosmic rays. Astrophys. J. 842 (1), 50. arXiv:1708.05013.

Ellison, D.C., Patnaude, D.J., Slane, P. \& Raymond, J. 2010 Efficient cosmic ray acceleration, hydrodynamics, and self-consistent thermal X-ray emission applied to supernova remnant RX J1713.7-3946. Astrophys. J. 712, 287-293. arXiv:1001.1932.

Evoli, C., Aloisio, R. \& Blasi, P. 2019 Galactic cosmic rays after the AMS-02 observations. Phys. Rev. D 99 (10), 103023. arXiv:1904.10220.

Evoli, C., Blasi, P., Amato, E. \& Aloisio, R. 2020a Signature of energy losses on the cosmic ray electron spectrum. Phys. Rev. Lett. 125 (5), 051101. arXiv:2007.01302.

Evoli, C., Morlino, G., Blasi, P. \& Aloisio, R. $2020 b$ AMS-02 beryllium data and its implication for cosmic ray transport. Phys. Rev. D 101 (2), 023013. arXiv:1910.04113.

FERRAND, G. \& MARCOWITH, A. 2010 On the shape of the spectrum of cosmic rays accelerated inside superbubbles. Astron. Astrophys. 510, A101. arXiv:0911.4457.

Fukui, Y., Sano, H., Sato, J., Torit, K., Horachi, H., HayaKaWA, T., McClure-Griffiths, N.M., Rowell, G., Inoue, T., InUtsukA, S., et al. 2012 A detailed study of the molecular and atomic gas toward the $\gamma$-ray supernova remnant RX J1713.7-3946: spatial TeV $\gamma$-ray and interstellar medium gas correspondence. Astrophys. J. 746, 82. arXiv:1107.0508.

FUnK, S. 2015 Ground- and space-based gamma-ray astronomy. Annu. Rev. Nucl. Part. Sci. 65, $245-277$. arXiv:1508.05190. 
Gabici, S. \& Aharonian, F.A. 2014 Hadronic gamma-rays from RX J1713.7-3946? Mon. Not. R. Astron. Soc. 445, L70-L73. arXiv:1406.2322.

Gabici, S., Aharonian, F.A. \& Blasi, P. 2007 Gamma rays from molecular clouds. Astrophys. Space Sci. 309, 365-371. arXiv:astro-ph/0610032.

Gabici, S., Aharonian, F.A. \& CASANOVA, S. 2009 Broad-band non-thermal emission from molecular clouds illuminated by cosmic rays from nearby supernova remnants. Mon. Not. R. Astron. Soc. 396 (3), 1629-1639. arXiv:0901.4549.

Gaggero, D., Grasso, D., Marinelli, A., Taoso, M. \& Urbano, A. 2017 Diffuse cosmic rays shining in the galactic center: a novel interpretation of H.E.S.S. and fermi-LAT $\gamma$-ray data. Phys. Rev. Lett. 119 (3), 031101. arXiv:1702.01124.

Gaggero, D., Maccione, L., Di Bernardo, G., Evoli, C. \& Grasso, D. 2013 Three-dimensional model of cosmic-ray lepton propagation reproduces data from the alpha magnetic spectrometer on the international space station. Phys. Rev. Lett. 111, 021102.

Ghez, A.M., Salim, S., Weinberg, N.N., Lu, J.R., Do, T., Dunn, J.K., Matthews, K., MorRIS, M.R., YELDA, S., BECKLIN, E.E., et al. 2008 Measuring distance and properties of the Milky Way's central supermassive black hole with stellar orbits. Astrophys. J. 689 (2), 1044-1062. arXiv:0808.2870.

GiacAlone, J. \& JOKIPII, J.R. 2007 Magnetic field amplification by shocks in turbulent fluids. Astrophys. J. Lett. 663 (1), L41-L44.

Gillessen, S., Eisenhauer, F., Trippe, S., Alexander, T., Genzel, R., Martins, F. \& Ott, T. 2009 Monitoring stellar orbits around the massive black hole in the galactic center. Astrophys. $J$. 692 (2), 1075-1109.

GinZBURG, V.L. \& SYROVATSKII, S.I. 1964 The Origin of Cosmic Rays. Elsevier.

Giuliani, A., Cardillo, M., Tavani, M., Fukui, Y., Yoshitke, S., Torit, K., Dubner, G., Castelletti, G., Barbiellini, G., Bulgarelli, A., et al. 2011 Neutral pion emission from accelerated protons in the supernova remnant W44. Astrophys. J. Lett. 742 (2), L30. arXiv:1111.4868.

Grießmeier, J.M., Tabataba-Vakili, F., Stadelmann, A., Grenfell, J.L. \& Atri, D. 2015 Galactic cosmic rays on extrasolar Earth-like planets. I. Cosmic ray flux. Astron. Astrophys. 581, A44. arXiv:1509.00735.

H.E.S.S. Collaboration, Abdalla, H., Abramowski, A., Aharonian, F., Benkhali, F.A., Akhperjanian, A.G., Andersson, T., Angüner, E.O., Arrieta, M., Arrieta, P., et al. 2018 H.E.S.S. observations of RX J1713.7-3946 with improved angular and spectral resolution: evidence for gamma-ray emission extending beyond the X-ray emitting shell. Astron. Astrophys. 612, A6. arXiv: 1609.08671.

H.E.S.S. Collaboration, Abramowski, A., Aharonian, F., Benkhali, F.A., Akhperjanian, A.G., Angüner, E.O., BAckes, M., Balenderan, S., Balzer, A., Barnacka, A., et al. 2015 The exceptionally powerful $\mathrm{TeV} \gamma$-ray emitters in the large magellanic cloud. Science 347 (6220), 406-412. arXiv:1501.06578.

H.E.S.S. Collaboration, Abramowski, A., Aharonian, F., Benkhali, F.A., Akhperjanian, A.G., Angüner, E.O., Backes, M., Balzer, A., Becherini, Y., TuUs, J., et al. 2016 Acceleration of petaelectronvolt protons in the galactic centre. Nature 531 (7595), 476-479. arXiv:1603.07730.

Heyer, M. \& Dame, T.M. 2015 Molecular clouds in the Milky Way. Annu. Rev. Astron. Astrophys. $53,583-629$.

Higdon, J.C. \& LingENFELTER, R.E. 2005 OB associations, supernova-generated superbubbles, and the source of cosmic rays. Astrophys. J. 628 (2), 738-749.

Holcomb, C. \& SPITKOVSKY, A. 2019 On the growth and saturation of the gyroresonant streaming instabilities. Astrophys. J. 882 (1), 3. arXiv:1811.01951.

Hona, B., Fleischiack, H. \& Huentemeyer, P. 2019 Testing the limits of particle acceleration in Cygnus OB2 with HAWC. PoS, ICRC2019, p. 699. PoS - Proceedings of Science.

Ibarra, A., Tran, D. \& Weniger, C. 2013 Indirect searches for decaying dark matter. Intl J. Mod. Phys. A 28 (27), 1330040. arXiv:1307.6434. 
Inoue, T., Yamazaki, R., InUtsuka, S.-I. \& FukUi, Y. 2012 Toward understanding the cosmic-ray acceleration at young supernova remnants interacting with interstellar clouds: possible applications to RX J1713.7-3946. Astrophys. J. 744, 71. arXiv:1106.3080.

IsSA, M.R. \& Wolfendale, A.W. 1981 Gamma rays from the cosmic ray irradiation of local molecular clouds. Nature 292, 430-433.

Kafexhiu, E., Aharonian, F., Taylor, A.M. \& Vila, G.S. 2014 Parametrization of gamma-ray production cross sections for $p p$ interactions in a broad proton energy range from the kinematic threshold to PEV energies. Phys. Rev. D 90, 123014.

Kelner, S.R., Aharonian, F.A. \& Bugayov, V.V. 2006 Energy spectra of gamma rays, electrons and neutrinos produced at proton-proton interactions in the very high energy regime. Phys. Rev. D 74, 034018 .

KlePach, E.G., Ptuskin, V.S. \& Zirakashvili, V.N. 2000 Cosmic ray acceleration by multiple spherical shocks. Astropart. Phys. 13, 161-172.

Konopelko, A., Atkins, R.W., Blaylock, G., Buckley, J.H., Butt, Y., Carter-Lewis, D.A., CElik, O., Cogan, P., ChOW, Y.C.K., CuI, W., et al. 2007 Observations of the unidentified TeV $\gamma$-ray source TeV J2032+4130 with the Whipple Observatory $10 \mathrm{~m}$ telescope. Astrophys. J. 658, 1062-1068. arXiv:astro-ph/0611730.

Korsmeier, M., Donato, F. \& Di Mauro, M. 2018 Production cross sections of cosmic antiprotons in the light of new data from the NA61 and LHCb experiments. Phys. Rev. D 97 (10), 103019. arXiv: 1802.03030.

Lagage, P.O. \& CeSARSKy, C.J. 1983 The maximum energy of cosmic rays accelerated by supernova shocks. Astron. Astrophys. 125, 249-257.

Lazarian, A., Eyink, G.L., Jafari, A., Kowal, G., Li, H., XU, S. \& Vishniac, E.T. 2020 3D turbulent reconnection: theory, tests, and astrophysical implications. Phys. Plasmas 27 (1), 012305. arXiv:2001.00868.

LIPARI, P. 2017 Interpretation of the cosmic ray positron and antiproton fluxes. Phys. Rev. D 95 (6), 063009. arXiv:1608.02018.

Malone, K. \& HAWC Collaboration 2017 The gamma-ray sky above $50 \mathrm{TeV}$ with the HAWC observatory. In APS April Meeting Abstracts, p. X4.008.

Montmerle, T. \& Cesarsky, C. 1979 Gamma-ray emission from snobs. In International Cosmic Ray Conference, vol. 1, p. 191. Institute for Cosmic Ray Research, University of Tokyo.

Morlino, G. \& AMATO, E. 2020 Impact of transport modeling on the ${ }^{6} 0 \mathrm{Fe}$ abundance inside galactic cosmic ray sources. Phys. Rev. D 101 (8), 083017. arXiv:2003.04700.

Morlino, G., Amato, E. \& Blasi, P. 2009 Gamma-ray emission from SNR RX J1713.7-3946 and the origin of galactic cosmic rays. Mon. Not. R. Astron. Soc. 392 (1), 240-250. arXiv:0810.0094.

MorRison, P. 1957 On the origins of cosmic rays. Rev. Mod. Phys. 29, 235-243.

Moskalenko, I.V. \& Strong, A.W. 1998 Production and propagation of cosmic-ray positrons and electrons. Astrophys. J. 493 (2), 694-707. arXiv:astro-ph/9710124.

MurraY, N. 2011 Star formation efficiencies and lifetimes of giant molecular clouds in the Milky Way. Astrophys. J. 729 (2), 133.

Nava, L., Gabici, S., Marcowith, A., Morlino, G. \& Ptuskin, V.S. 2016 Non-linear diffusion of cosmic rays escaping from supernova remnants - I. The effect of neutrals. Mon. Not. R. Astron. Soc. 461 (4), 3552-3562. arXiv:1606.06902.

OHIRA, Y. 2016 Magnetic field amplification by collisionless shocks in partially ionized plasmas. Astrophys. J. 817 (2), 137. arXiv:1512.04167.

Oka, T., Hasegawa, T., Sato, F., Tsuboi, M. \& Miyazaki, A. 1998 A large-scale CO survey of the galactic center. Astrophys. J. Suppl. Ser. Astrophys. J. Suppl. Ser. 118 (2), 455-515.

Padovani, M., Ivlev, A.V., Galli, D., OfFner, S.S.R., Indriolo, N., Rodgers-Lee, D., Marcowith, A., Girichidis, P., Bykov, A.M. \& Kruijssen, J.M.D. 2020 Impact of low-energy cosmic rays on star formation. Space Sci. Rev. 216 (2), 29. arXiv:2002.10282.

Parizot, E., Marcowith, A., van der Swaluw, E., Bykov, A.M. \& Tatischeff, V. 2004 Superbubbles and energetic particles in the Galaxy. I. Collective effects of particle acceleration. Astron. Astrophys. 424, 747-760. arXiv:astro-ph/0405531. 
Particle Data Group, Zyla, P.A., Barnett, R.M., Beringer, J., Dahl, O., Dwyer, D.A., Groom, D.E., Lin, C.J., Lugovsky, K.S., PiAnOri, E., et al. 2020 Review of particle physics. Prog. Theor. Expl Phys. 2020 (8), 083C01.

Peron, G., Aharonian, F., Casanova, S., Zanin, R. \& Romoli, C. 2020 On the gamma-ray emission of w44 and its surroundings. Astrophys. J. 896 (2), L23.

PfeffermanN, E. \& Aschenbach, B. 1996 Rosat observation of a new supernova remnant in the constellation scorpius. In International Conference on X-ray Astronomy and Astrophysics: Röntgenstrahlung from the Universe, -1, 267-268.

Pohl, M., YAn, H. \& LAZARian, A. 2005 Magnetically limited X-ray filaments in young supernova remnants. Astrophys. J. Lett. 626 (2), L101-L104.

Recchia, S., Gabici, S., Aharonian, F.A. \& Vink, J. 2019 Local fading accelerator and the origin of TeV cosmic ray electrons. Phys. Rev. D 99 (10), 103022. arXiv:1811.07551.

Reid, M.J., Menten, K.M., Brunthaler, A., Zheng, X.W., Dame, T.M., Xu, Y., Wu, Y., ZhANG, B., SANNA, A., SATO, M., et al. 2014 Trigonometric parallaxes of high mass star forming regions: the structure and kinematics of the Milky Way. Astrophys. J. 783 (2), 130. arXiv:1401.5377.

Reimer, A., Pohl, M. \& Reimer, O. 2006 Nonthermal high-energy emission from colliding winds of massive stars. Astrophys. J. 644 (2), 1118-1144. arXiv:astro-ph/0510701.

RetTig, R. \& PohL, M. 2012 The properties of non-thermal X-ray filaments in young supernova remnants. Astron. Astrophys. 545, A47. arXiv:1208.5322.

Reville, B., O'Sullivan, S., Duffy, P. \& Kirk, J.G. 2008 The transport of cosmic rays in self-excited magnetic turbulence. Mon. Not. R. Astron. Soc. 386 (1), 509-515. arXiv:0802.0109.

Riquelme, M.A. \& Spitkovsky, A. 2009 Nonlinear study of Bell's cosmic ray current-driven instability. Astrophys. J. 694 (1), 626-642. arXiv:0810.4565.

RogachevskiI, I., Kleeorin, N., Brandenburg, A. \& Eichler, D. 2012 Cosmic-ray current-driven turbulence and mean-field dynamo effect. Astrophys. J. 753 (1), 6. arXiv:1204.4246.

Roman-Duval, J., Jackson, J.M., Heyer, M., Johnson, A., RAthborne, J., Shah, R. \& Simon, R. 2009 Kinematic distances to molecular clouds identified in the galactic ring survey. Astrophys. $J$. 699 (2), 1153-1170. arXiv:0905.0723.

Schure, K.M. \& Bell, A.R. 2014 From cosmic ray source to the Galactic pool. Mon. Not. R. Astron. Soc. 437 (3), 2802-2805. arXiv:1310.7027.

Schure, K.M., Bell, A.R., Drury, L.O'C. \& Bykov, A.M. 2012 Diffusive shock acceleration and magnetic field amplification. Space Sci. Rev. 173 (1-4), 491-519. arXiv:1203.1637.

Shikaze, Y., Haino, S., Abe, K., Fuke, H., Hams, T., Kim, K.C., Makida, Y., Matsuda, S., Mitchell, J.W., Moiseev, A.A., et al. 2007 Measurements of $0.220 \mathrm{GeV} / \mathrm{n}$ cosmic-ray proton and helium spectra from 1997 through 2002 with the BESS spectrometer. Astropart. Phys. 28 (1), 154-167. arXiv:astro-ph/0611388.

SkILLING, J. 1975 Cosmic ray streaming - II. Effect of particles on Alfvén waves. Mon. Not. R. Astron. Soc. $173,245-254$.

Stone, E.C., Cummings, A.C., McDonald, F.B., Heikkila, B.C., Lal, N. \& Webber, W.R. 2013 Voyager 1 observes low-energy galactic cosmic rays in a region depleted of heliospheric ions. Science 341 (6142), 150-153.

Sveshnikova, L.G., Strelnikova, O.N. \& Ptuskin, V.S. 2013 Spectrum and anisotropy of cosmic rays at TeV-PeV-energies and contribution of nearby sources. Astropart. Phys. 50, 33-46. arXiv:1301.2028.

Tanaka, T., Uchiyama, Y., Aharonian, F.A., Takahashi, T., Bamba, A., Hiraga, J.S., Kataoka, J., Kishishita, T., Kokubun, M., Mori, K., et al. 2008 Study of nonthermal emission from SNR RX J1713.7-3946 with Suzaku. Astrophys. J. 685, 988-1004. arXiv:0806.1490.

Tavani, M., Giuliani, A., Chen, A.W., Argan, A., Barbiellini, G., Bulgarelli, A., Caraveo, P., Cattaneo, P.W., Cocco, V., Contessi, T., et al. 2010 Direct evidence for hadronic cosmic-ray acceleration in the supernova Remnant IC 443. Astrophys. J. Lett. 710 (2), L151-L155. arXiv:1001.5150.

THOUdAM, S. \& HöRANDEL, J.R. 2012 Nearby supernova remnants and the cosmic ray spectral hardening at high energies. Mon. Not. R. Astron. Soc. 421 (2), 1209-1214. arXiv:1112.3020. 
TomassetTi, N. 2012 Origin of the cosmic-ray spectral hardening. Astrophys. J. Lett. 752 (1), L13. arXiv: 1204.4492.

Torres, D.F., Domingo-Santamaría, E. \& Romero, G.E. 2004 High-energy gamma rays from stellar associations. Astrophys. J. Lett. 601 (1), L75-L78. arXiv:astro-ph/0312128.

Tsuboi, M., Handa, T. \& UKitA, N. 1999 Dense molecular clouds in the galactic center region. I. Observations and data. Astrophys. J. Suppl. Ser. 120, 1-39.

Uchiyama, Y., Aharonian, F.A., Tanaka, T., Takahashi, T. \& Maeda, Y. 2007 Extremely fast acceleration of cosmic rays in a supernova remnant. Nature 449 (7162), 576-578.

Uchiyama, Y., Blandford, R.D., Funk, S., Tajima, H. \& Tanaka, T. 2010 Gamma-ray emission from crushed clouds in supernova remnants. Astrophys. J. Lett. 723 (1), L122-L126. arXiv: 1008.1840 .

Uchiyama, Y., Funk, S., Katagiri, H., Katsuta, J., Lemoine-Goumard, M., Tajima, H., TANAKA, T. \& TORRES, D.F. 2012 Fermi large area telescope discovery of GeV gamma-ray emission from the vicinity of SNR W44. Astrophys. J. Lett. 749 (2), L35. arXiv:1203.3234.

VINK, J. 2012 Supernova remnants: the X-ray perspective. Astron. Astrophys. Rev. 20, 49. arXiv:1112.0576.

VoelK, H.J. \& Forman, M. 1982 Cosmic rays and gamma-rays from OB stars. Astrophys. J. 253, 188-198.

WANG, Z.-R., QU, Q.-Y. \& CHEN, Y. 1997 Is RX j1713.7-3946 the remnant of the AD393 guest star? Astron. Astrophys., 318, L59-L61.

WentZel, D.G. 1974 Cosmic-ray propagation in the Galaxy: collective effects. Astron. Astrophys. Rev. 12, 71-96.

Yanasak, N.E., Wiedenbeck, M.E., Mewaldt, R.A., Davis, A.J., Cummings, A.C., George, J.S., Leske, R.A., Stone, E.C., Christian, E.R., von Rosenvinge, T.T., et al. 2001 Measurement of the secondary radionuclides ${ }^{1} 0 \mathrm{Be},{ }^{2} 6 \mathrm{Al},{ }^{3} 6 \mathrm{Cl},{ }^{5} 4 \mathrm{Mn}$, and ${ }^{1} 4 \mathrm{C}$ and implications for the galactic cosmic-ray age. Astrophys. J. 563 (2), 768-792.

YIN, P.-F., YU, Z.-H., YUAN, Q. \& BI, X.-J. 2013 Pulsar interpretation for the AMS-02 result. Phys. Rev. D 88, 023001.

ZABALZA, V. 2015 naima: a Python package for inference of relativistic particle energy distributions from observed nonthermal spectra. In Proceedings of International Cosmic Ray Conference 2015, p. 922. arXiv:1509.03319.

ZIRAKASHVILI, V.N. \& AHARONIAN, F.A. 2010 Nonthermal radiation of young supernova remnants: the case of RX J1713.7-3946. Astrophys. J. 708, 965-980. arXiv:0909.2285.

ZweIBEL, E.G. 2013 The microphysics and macrophysics of cosmic rays. Phys. Plasmas 20 (5), 055501. 